\title{
Insights into brine dynamics and sea ice desalination from a 1-D model study of gravity drainage
}

\author{
Philipp J. Griewank ${ }^{1,2}$ and Dirk Notz ${ }^{1}$ \\ Received 13 September 2012; revised 22 April 2013; accepted 20 May 2013; published 15 July 2013.
}

[1] We study gravity drainage using a new 1-D, multiphase sea ice model. A parametrization of gravity drainage based on the convective nature of gravity drainage is introduced, whose free parameters are determined by optimizing model output against laboratory measurements of sea ice salinity evolution. Optimal estimates of the free parameters as well as the parametrization performance remain stable for vertical grid resolutions from 1 to $30 \mathrm{~mm}$. We find a strong link between sea ice growth rate and bulk salinity for constant boundary conditions but only a weak link for more realistic boundary conditions. We also demonstrate that surface warming can trigger brine convection over the whole ice layer. Over a growth season, replacing the convective parametrization with constant initial salinities leads to an overall 3\% discrepancy of stored energy, thermal resistance, and salt release. We also derive from our convective parametrization a simplified, numerically cheap and stable gravity-drainage parametrization. This parametrization results in an approximately $1 \%$ discrepancy of stored energy, thermal resistance, and salt release compared to the convective parametrization. A similarly low discrepancy to our complex parametrization can be reached by simply prescribing a depthdependent salinity profile.

Citation: Griewank, P. J., and D. Notz (2013), Insights into brine dynamics and sea ice desalination from a 1-D model study of gravity drainage, J. Geophys. Res. Oceans, 118, 3370-3386, doi:10.1002/jgrc.20247.

\section{Introduction}

[2] Gravity drainage, which is the convective exchange of cold and dense brine with fresher seawater, is the dominant desalination process in sea ice [Notz and Worster, 2006, 2009] and plays a crucial role in sea ice biogeochemistry by replenishing the ice with nutrients [Vancoppenolle et al., 2010]. Gravity drainage can also be used to efficiently desalinate sea water [Gu et al., 2012]. In this paper, we study gravity drainage using the newly developed 1-D thermodynamic sea ice model SAMSIM (semiadaptive multiphase sea-ice model) with a convective gravitydrainage parametrization. The model is used in particular to quantify how gravity drainage affects the thermodynamic properties of sea ice. We also present a simplified salinity parametrization based on our convective parametrization that is suitable for climate models.

[3] Our current understanding of gravity drainage is far from complete, partly because detailed measurements of

\footnotetext{
${ }^{1}$ Max Planck Institute for Meteorology, Hamburg, Germany.

${ }^{2}$ International Max Planck Research School on Earth System Modelling, Hamburg, Germany.

Corresponding author: P. J. Griewank, Max Planck Institute for Meteorology, Bundesstr. 53, DE-20146 Hamburg, Germany. (philipp.griewank@zmaw.de).
}

(C)2013. American Geophysical Union. All Rights Reserved. 2169-9275/13/10.1002/jgrc.20247 brine flow in sea ice are largely lacking. Most of our current understanding stems from evaluating salinity measurements from ice cores and laboratory studies of growing multiphase materials [e.g., Chen, 1995; Wettlaufer et al., 1997; Cottier et al., 1999]. However, growing and measuring sea ice in the laboratory over many weeks are practical challenges, and (to our knowledge) no laboratory sea ice experiments lasting longer than a month have been conducted.

[4] Detailed field studies of growing sea ice through icecore series are rare owing to the severe logistical issues of taking and processing ice cores under inhospitable climate conditions. Hence, only few such studies exist, most notably are those conducted by Nakawo and Sinha [1981], Lei et al. [2010], and Gough et al. [2012]. Unfortunately, measuring salinity by ice cores has many drawbacks. These include brine loss from cores, low temporal resolution, and the inability to sample repeatedly due to the destructive nature of core extraction. Gough et al. [2012] conducted a very thorough analysis of their core data showcasing that multiple cores are necessary to obtain representative values. This is due to the high horizontal variability of sea ice, and because salinity measurements from the same core at different heights cannot be treated as independent due to a high vertical correlation of measured salinity anomalies.

[5] In this paper, we study gravity drainage numerically. Previous numerical studies can be split into 2-D approaches, which simulate the flow field of brine in a vertical slice of growing sea ice, and 1-D approaches, which 
parametrize the brine flow and its effects on the vertical sea ice profile. The 2-D models have the drawback of being computationally expensive and/or limited to well-defined test cases [see Oertling and Watts, 2004; Petrich et al., 2004; Wells et al., 2010]. Proposed 1-D parametrizations are either based on the quantitative estimates of Cox and Weeks [1988], or treat gravity drainage as a diffusive process similar to turbulent diffusion in a mixed layer [Vancoppenolle et al., 2010; Jeffery et al., 2011]. However, both of these 1-D methods are inconsistent with laboratory experiments and 2-D simulations from which we know that gravity drainage is not a turbulent process. Saenz and Arrigo [2012] were the first to take the convective nature of gravity drainage partially into account, but their gravitydrainage parametrization is still based on the simplified estimates of Cox and Weeks [1988]. Our approach extends the findings of small-scale laboratory experiments and 2-D numerical simulations to large and longer scales using a 1-D thermodynamic model based on mushy-layer theory and a convective gravity-drainage parametrization derived from research on brine fluxes from solidifying binary alloys [Wells et al., 2010]. A key property of the newly developed thermodynamic multiphase model SAMSIM is a semiadaptive grid, which gives us an advantage over previous attempts to parametrize gravity drainage. Instead of prescribing an explicit ice-ocean front, as in the Maykut and Untersteiner [1971] model and all its descendants [e.g., Semtner, 1976; Bitz and Lipscomb, 1999; Huwald et al., 2005a], the grid ensures that the ice-ocean interface is always well approximated without imposing any assumptions of salinity, temperature, or growth rate. Open questions we address in this paper are the link between sea ice growth speed and bulk salinity, whether gravity drainage can penetrate deep into the ice, and how gravity drainage can be represented in climate models.

[6] Our new convective parametrization is ill-suited for earth system models as it requires a small time step to avoid instabilities. As an alternative we derive a simpler parametrization from the convective parametrization which can improve sea ice thermodynamics and salt release into the ocean for climate models. In this paper we refer to the simpler salinity parametrization as the simple parametrization and to the more complex parametrization that calculates brine fluxes as the convective parametrization.

[7] Section 2 provides a brief description of SAMSIM. In section 3 we introduce the full convective parametrization. Based on it, we also devise the simple salinity parametrization. Section 4 contains a description of the Levenberg-Marquadt optimization algorithm and data used to determine the free parameters of our parametrizations. In section 5 we conduct our first experiments using idealized boundary conditions. Here we study how growth speeds influence bulk salinity and how deep convection can be triggered. These findings are then compared to a more realistic growth season simulated by forcing the model with three-hourly ERA-reanalysis data in section 6. Using this growth season, we study how the thermal properties of the sea ice vary when the salinity is either prescribed, or simulated using the simple parametrization that we introduced in section 3. Finally, in section 7, we present a summary of our results and conclusions and discuss how gravity drainage can be represented in climate models.

\section{SAMSIM Description}

[8] In the following section, we provide a brief overview of SAMSIM. The thermodynamic core of SAMSIM is derived from the mushy-layer equations of sea ice [Feltham et al., 2006]. Our approach is similar to that of Notz and Worster [2006] but was extended to also include a gas phase and gravity drainage. For an in-depth discussion on multiphase sea ice models, see Hunke et al. [2011].

[9] In contrast to commonly used front-tracking models [see Maykut and Untersteiner, 1971; Semtner, 1976; Bitz and Lipscomb, 1999; Huwald et al., 2005a; Saenz and Arrigo, 2012], SAMSIM has no prescribed ice-ocean front. In a front-tracking method, ice grows by changing the position of the ice-ocean interface at each time step. In contrast, in SAMSIM the solid fraction increases in a grid layer which has a constant thickness at each time step. Although there are many reasons to prefer the front-tracking approach, our approach grants us some additional freedom which we exploit when parametrizing brine dynamics. Additionally, there is a simple theoretical elegance in directly representing sea ice and water as a continuum of varying solid fraction, consistent with the mushy-layer nature of sea ice.

[10] SAMSIM is a finite-volume model to allow simple conservation of all conserved properties, such as mass, energy, and tracers. Currently, the spatial and temporal discretization schemes that are used to solve the heat transport equation

$$
q=-k \frac{\partial T}{\partial z}
$$

are explicit and of first order. The time-integrated heat flux between the layer $i$ and $i+1$ over a time step of length $\mathrm{d} t$ is

$$
\int_{t}^{t+d t} q \mathrm{~d} t=-k \frac{T^{i}-T^{i-1}}{\frac{\Delta z^{i}+\Delta z^{i-1}}{2}} \cdot d t
$$

which requires a small time step to satisfy the CourantFriedrichs-Lewy condition for heat diffusion

$$
\frac{k \mathrm{~d} t}{\rho c \Delta z^{2}}<0.5
$$

[11] Higher-order and implicit schemes can be implemented if a longer time step is needed but were unnecessary for this study as we require a small time step to resolve the brine dynamics.

\subsection{Layer Properties}

[12] SAMSIM is a 1-D finite-volume model, in which each layer is horizontally and vertically homogeneous and all phases are in local thermal equilibrium with each other. Each layer is defined by four core variables: absolute salinity $S_{a b s}$, absolute enthalpy $H_{a b s}$, mass $m$, and thickness $\Delta z$. The absolute enthalpy is the total Joules of enthalpy, and the absolute salinity is grams of salt in the layer. From the absolute salinity, absolute enthalpy, and mass we derive temperature $T$ and solid mass fraction $\psi$ by numerically 
solving the following set of equations for enthalpy $(H)$, bulk salinity $\left(S_{b u}\right)$, brine salinity $\left(S_{b r}\right)$, and $\psi$ :

$$
\begin{gathered}
H=\frac{H_{a b s}}{m}=-\psi L+f(T) \\
S_{b u}=\frac{S_{a b s}}{m}=S_{b r}(1-\psi) \\
S_{b r}=g(T) .
\end{gathered}
$$

[13] The appropriate value of latent heat $(L)$, the integral of the heat capacity with respect to temperature $(f(T))$, and the brine salinity as a function of temperature $(g(T))$ are material specific, and their accuracy can be varied as desired. By approximating gas as massless, we can derive the solid, liquid, and gas phase volume fractions $\left(\phi_{s}, \phi_{l}\right.$, and $\phi_{g}$ ) from $\psi, \Delta z$, and $m$.

[14] Salt is treated as a massless tracer, but brine density is a function of brine salinity. When brine moves between layers, salt advection is calculated via the simple upstream method. The simple upstream method is artificially diffusive, especially when the tracer concentration has steep gradients. Since the brine salinity is determined by the temperature and since the temperature profile in sea ice is rather smooth, the artificial diffusion for salinity is small. If passive tracers were introduced, a more sophisticated advection method might be needed.

[15] The thermal conductivity of each layer is simply the volume-weighted sum of the solid and liquid fractions $k=\phi_{s} k_{s}+\phi_{l} k_{l}$. The gas fraction is treated as a perfect insulator and does not contribute to the layer's conductivity.

\subsection{Semiadaptive Grid}

[16] SAMSIM employs an irregular 1-D grid which we refer to as a semiadaptive grid for lack of a better term. This grid consists of a set number of top and bottom layers $\left(N_{\text {top }}\right.$ and $\left.N_{b o t}\right)$ with a constant thickness of $\Delta z_{0}$, and a variable number of adaptive middle layers $\left(N_{\text {mid }}\right)$ that grow and shrink in steps of $\Delta z_{0} / N_{\text {mid }}$ as needed. When the ice is so thin that not all layers are needed surplus layers are deactivated. When the number of active layers $(n)$ is less or equal to the maximum number of layers $\left(N=N_{\text {top }}+N_{\text {mid }}+\right.$ $\left.N_{\text {bot }}\right)$, all active layers share the thickness $\Delta z_{0}$. If no ice is present at all, SAMSIM shrinks to a single layer. The layers are indexed from top to bottom. This means that the index $i$ of the top layer is 1 , the lowest active layer has the index $n$, and when all layers are active the lowest layer has the index $N$.

[17] Figure 1 shows how SAMSIM's semiadaptive grid evolves during growth for $N=5, N_{t o p}=1, \quad N_{\text {mid }}=2$, $N_{b o t}=2$. Starting from a single layer of open water $(n=1)$, the grid grows to ensure that the solid volume fraction $\phi_{s}^{n}$ in the lowest active layer always lies below a certain fixed value $\left(\phi_{s}^{n}<\phi_{s}^{\mathrm{min}}\right)$. When $\phi_{s}^{n}$ increases beyond the limit value $\phi_{s}^{m i n}$ a new layer of underlying ocean water is added. If not all layers are activated $(n<N)$ the new layer is created by activating one of the previously deactivated layers. If $n=N$ then the uppermost bottom layer is merged into the middle layers, and all the bottom layers are shifted downward by one. When this occurs, all middle layers grow thicker by $\Delta z_{0} / N_{\text {mid }}$. The resulting changes of the core var-

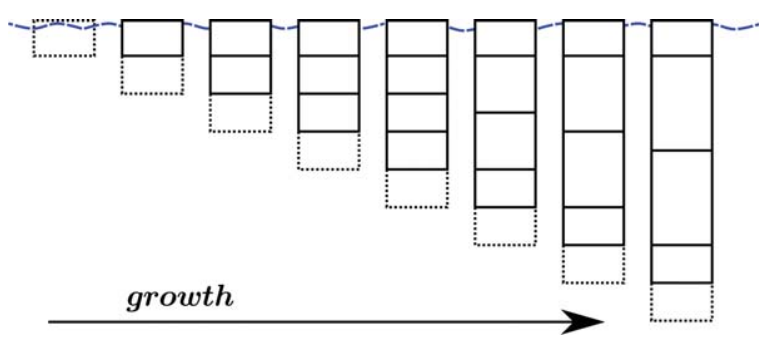

Figure 1. Semiadaptive grid evolution during growth for $N=5, N_{\text {top }}=1, N_{\text {mid }}=2, N_{\text {bot }}=2$ (see section 2.2).

iables in the middle layers are calculated using simple upstream advection. For example, if only two middle layers exist both layers grow by $\Delta z_{0} / 2$, and a mass of $\Delta z_{0} / 2$ times the density of the lower middle layers is reallocated to the upper middle layer. Conversely, the lowest layer is dissolved when $\phi_{s}^{n}=0$ and $\phi_{s}^{n-1}<\phi_{s}^{\min } / 2$. The lowest layer is dissolved only when $\phi_{s}^{n-1}<\phi_{s}^{\text {min }} / 2$ to ensure that new layers are not dissolved shortly after forming, when $\phi_{s}^{n}=0$ and $\phi_{s}^{n-1} \approx \phi_{s}^{\min }$. It is possible to set $\phi_{s}^{\min }$ to 0 , but under certain conditions, this can lead to many bottom layers with very low solid fractions. In nature, these very low solid fractions would indicate free-floating ice crystals. The parameter $\phi_{s}^{\mathrm{min}}$ can be understood physically as the minimum amount of ice needed for the ice crystals to form a connected mushy layer.

[18] All tests performed in this paper use a value of 0.05 for $\phi_{s}^{\text {min }}$, which results in a slight artificial cut off at vertical resolutions of 1 or $2 \mathrm{~mm}$. Raising $\phi_{s}^{\mathrm{min}}$ up to 0.10 barely effects results, but instabilities occurred at values lower than 0.03 .

[19] The semiadaptive grid has three major advantages. First, it allows SAMSIM to keep the spatial resolution constantly high at the ice-ocean and ice-atmosphere boundaries without exceeding a set maximum amount of layers. The second advantage is that no numerical diffusion occurs in the bottom layers due to moving layer boundaries. Instead, newly formed bottom layers retain their salinity, enthalpy, and mass as they are shifted upward in steps until they are merged into the middle layers. The final advantage is that the lowest layer-which represents the water at the iceocean interface - can evolve freely, which lets SAMSIM imitate processes such as underplating to a certain extent. Underplating refers to the collection of relatively light freshwater below the ice and above the denser underlying ocean water.

[20] For the aims of this study, these advantages of the semiadaptive grid far outweigh its disadvantages. These disadvantages include temporal discontinuities in the simulations caused by the finite-size, stepwise addition, and removal of layers. Additionally, vertical tracer advection across the transition from thin to thicker layers can cause nonphysical tracer transport. However, these numerical artifacts are small and can safely be neglected in this paper, since gravity drainage is mostly localized to the thin bottom layers. A further disadvantage of SAMSIM's grid is possible difficulties in its horizontal advection, which, again, is irrelevant for our 1-D study. Finally, SAMSIM's grid causes a somewhat larger computational burden compared to traditional grids, because the thin top and bottom layers limit the time step. 
[21] For specific purposes, such as calculating the ice thickness, we require a defined ice-ocean front which is not provided a priori by SAMSIM's grid. For such purposes we linearly interpolate a value from the solid volume fraction of the lowest layer. For example, if $\phi_{s}^{n}=\phi_{s}^{\min } / 3$, we would assume the upper third of the bottom layer to contain sea ice. The diagnosed ice-ocean front does not move smoothly, especially during melt. The impact of these steps on the Rayleigh number is discussed in section 5.2.

[22] For the purpose of this paper, snow is treated as a single layer of varying thickness with constant density and constant thermal conductivity. Although this simple setup is still standard for sea ice components of earth system models, there have been recent efforts to include more sophisticated representations of snow in climate models since the snow has such a low and varying thermal conductivity [Lecomte et al., 2011].

\subsection{Brine Expulsion}

[23] Because the density of ice is lower than that of water, freezing sea ice expels excess brine. This process is known as brine expulsion and was once believed to be an important desalination process in thin ice [Cox and Weeks, 1975]. Notz and Worster [2006, 2009] have demonstrated that although brine expulsion redistributes salt in the sea ice, the amount of salt that leaves the ice is negligibly small. However, brine expulsion is crucial to the density evolution of sea ice.

[24] SAMSIM determines the amount of brine which is expelled by checking if the summed volume of liquid brine and solid ice exceeds the volume of the layer at each time step. If the volume does exceed the layer volume, SAMSIM assumes that the excess brine is always moved to the layer below, regardless of the properties of the lower layers. The same approach was used in the 1-D model of Maksym and Jeffries [2000]. In reality, brine can move upward as well. Upward displaced brine can cause thin skins of extremely salty brine on top of the sea ice, a behavior Roscoe et al. [2011] captured with time-lapse photography. However, since it has been shown analytically and numerically that the total amount of salt transported downward by expulsion leads to maximum deviations of roughly 1 ppt [Chiareli and Worster, 1995; Notz, 2005] and the amount of upward displaced brine is likely much smaller than the amount of downward displaced brine, we expect the bulk salinity errors in SAMSIM introduced by our unidirectional implementation of brine expulsion to be far below 1 ppt.

\section{Gravity-Drainage Parametrizations}

[25] In contrast to 2-D or 3-D models, a 1-D model is incapable of resolving a convective process, and gravity drainage can only be parametrized. Previous 1-D parametrizations of gravity drainage were presented by Cox and Weeks [1988], Vancoppenolle et al. [2010], Jeffery et al. [2011], and Saenz and Arrigo [2012]. The empirical approach of Cox and Weeks [1988] calculates desalination in growing ice as a combination of initial salt entrapment, brine expulsion, and gravity drainage. However, we know now from experiments and theory that both initial salt entrapment and brine expulsion do not desalinate the ice
[Notz and Worster, 2009]. Both Vancoppenolle et al. [2010] and Jeffery et al. [2011] treat gravity drainage as a diffusion caused by brine mixing, similar to turbulent mixing in boundary layers. However, both of these approaches are in contrast to studies of growing mushy layers which have shown that gravity drainage is a convective process linked to chimney formation [e.g., Tait and Jaupart, 1992; Chen, 1995; Wettlaufer et al., 1997; Notz and Worster, 2008]. In sea ice, these chimneys are commonly referred to as brine channels. Saenz and Arrigo [2012] were the first to incorporate some limited convective aspects of gravity drainage into a 1-D parametrization. However, the parametrization of Saenz and Arrigo [2012] relies heavily on empirical values, to determine both initial desalination and stable solid fractions. We have developed two new 1-D parametrizations of gravity drainage: a convective parametrization and derived from it, a simple parametrization. The convective parametrization attempts to simulate brine movement as accurately as possible based on a few core assumptions. The simple parametrization is an attempt to produce a realistic salinity evolution at a lower computational cost.

\subsection{Rayleigh Number}

[26] Following previous studies [e.g., Tait and Jaupart, 1992; Wettlaufer et al., 1997], the onset and strength of gravity drainage in our parametrizations are linked to a porous medium/mushy-layer Rayleigh number $(R)$. In general, such a Rayleigh number describes the ratio of driving buoyancy to both thermal diffusion and viscous resistance in a porous medium. However, the specific formulations used to calculate $R$ vary considerably and are highly dependent on the assumed permeability. Due to this high variability in definitions, it is difficult to compare Rayleigh number values from different studies. A clear distinction should be made between Rayleigh numbers that represent the whole vertical sea ice profile and discretized local Rayleigh numbers that represent the convective flow from a specific single horizontal layer to the underlying ocean. We use $R^{i}$ to refer to the Rayleigh number of the layer $i$. As many of our assumptions are based on the results of Wells et al. [2010], we strive to keep our definition of the Rayleigh number qualitatively similar to their definition.

[27] The parameter $R^{i}$ can be regarded as the ratio of two representative timescales: the advective timescale $t_{A}^{i}$ and the diffusive timescale $t_{D}^{i}$. The advective timescale is defined by the amount of time that the buoyancy driven brine in layer $i$ needs to reach the ice-ocean interface. According to Darcy's law the brine moves at a characteristic speed of

$$
v=\frac{g \Delta \rho \Pi}{\mu}
$$

in which $g$ is the gravitational acceleration, $\Delta \rho$ is the density difference between the brine and the underlying ocean water, $\mu$ is the dynamic viscosity of the brine, and $\Pi$ is the sea ice permeability which is discussed in section 3.4. Accordingly, the time needed for brine to move the distance $h^{i}$ from layer $i$ to the ice-ocean interface equals 


$$
t_{A}^{i}=\frac{h^{i} \mu}{\tilde{\Pi}^{i} g \Delta \rho^{i}} .
$$

[28] Instead of the permeability of the layer $i$ we use the minimal permeability of the layers beneath $i$,

$$
\tilde{\Pi}^{i}=\min \left(\Pi^{i}, \Pi^{i+1}, \ldots, \Pi^{n}\right)
$$

as the most impermeable layer acts as a bottleneck to the flow. Using the minimal permeability is a simplification of the harmonic mean, which is the correct approach to determine the bulk permeability for a Darcy flow through a stack of layers.

[29] The diffusive timescale

$$
t_{D}^{i}=\frac{\left(h^{i}\right)^{2}}{\kappa}
$$

represents the diffusion time of thermal anomalies over the distance $h^{i}$ for a given thermal diffusivity $\kappa$. The diffusive timescale reflects the time necessary for the relatively cold brine traveling downward in the channels to warm to the temperature of the surrounding sea ice.

[30] To calculate the thermal diffusivity, representative values of thermal conductivity, thermal capacity, and density must be chosen $\kappa=k /(\rho c)$. Often, the phase-averaged values of sea ice are chosen in accordance with mushylayer theory [e.g., Vancoppenolle et al., 2006; Wells et al., 2010, 2011]. However, as mushy-layer theory is based on the phase liquidus relation at thermal equilibrium, it cannot capture the nonequilibrium thermal interactions between the cold brine in the channels and the warmer surrounding sea ice. The heat flux from the sea ice to the brine channels depends on the geometry of the brine channels, the flow field in the channels, and the speed with which the salty brine dissolves the channel walls. Instead of the phaseaveraged values of sea ice, we use the thermal conductivity, capacity, and density of the brine to calculate the thermal diffusivity for the following reasons. First, due to the fact that the mass of the surrounding sea ice is much greater than the mass of the brine flowing through the channels, the surrounding sea ice cools much less than the brine in the channels warms and can be considered thermally inert. Second, as the thermal diffusivity of the brine in the channels is up to eight times smaller than the phase-averaged values of the surrounding sea ice, thermal anomalies in the brine channel persist longer than in the surrounding sea ice. Both of these considerations indicate that the speed with which the cold brine in the channels approaches that of the surrounding sea ice is limited by the thermal diffusivity of the brine itself and not by the thermal diffusivity of the surrounding sea ice. Our reasoning focuses on the thermal imbalance in the brine channels, but it is possible that the thermal dissipation of the upwelling brine in the surrounding mush dominates. However, until a more detailed 2-D or 3-D analysis of gravity drainage can conclusively resolve the issue, we will use the brine thermal diffusivity as a working assumption.

[31] By computing density differences via the difference of brine salinity to the salinity of the lowest active layer $n$ which represents the water at the ice-ocean interface,
$\Delta \rho^{i}=\rho_{l} \beta \Delta S^{i}=\rho_{l} \beta \Delta\left(S_{b r}^{i}-S_{b r}^{n}\right)$, the resulting Rayleigh number is

$$
R^{i}=\frac{t_{D}^{i}}{t_{A}^{i}}=\frac{g \Delta \rho^{i} \tilde{\Pi}^{i} h^{i}}{\kappa \mu}=\frac{g \rho_{l} \beta \Delta S^{i} \tilde{\Pi}^{i} h^{i}}{\kappa \mu} .
$$

[32] A high Rayleigh number indicates that the moving brine flows quicker than thermal diffusion can enforce thermal equilibrium. As long as the moving brine is colder than the surrounding brine, it remains saltier and heavier and keeps descending. A low Rayleigh number indicates that thermal diffusion acts quicker than advection, returning the brine to thermal equilibrium and negating its buoyancy. Assuming both timescales are identical, brine in the ice would be brought into thermal (and salinity) equilibrium just as quick as it moves, resulting in a neutral buoyancy. This dependence of the convective strength on the Rayleigh number is the core of the convective parametrization we now turn to.

\subsection{Convective Parametrization}

[33] The convective parametrization strives to simulate the convective brine fluxes as accurately as possible. Our approach was heavily inspired by the 2-D numerical studies of growing mushy layers conducted by Petrich et al. [2004] and Wells et al. [2010]. By assuming that chimney spacing in growing mushy layers maximizes potential energy transport, Wells et al. [2010] linked solute flux to the Rayleigh number of the convecting mushy layer. They concluded that the solute flux increases approximately linearly with the Rayleigh number when the Rayleigh number is above a critical value. Below that value the circulation breaks down. Among the findings a recent study of solute fluxes through chimneys by D. J. Rees Jones and M. G. Worster (Fluxes through steady chimneys in a mushy layer during binary alloy solidification, submitted to Journal of Fluid Mechanics, 2012) is an analytically derived linear relationship of solute flux to Rayleigh number for 2-D planar flows. Rees Jones and Worster (submitted manuscript, 2012) numerically extended their approach to 3-D flows to discover some nonlinear behavior between solute flux and Rayleigh number. However, despite these nonlinear effects Rees Jones and Worster [2013] still recommend parametrizing gravity drainage using a linear relationship of Rayleigh number to solute flux.

[34] Wells et al. [2010] imitate a growing mushy layer with constant and well-defined boundaries using a quasisteady-state approach. As SAMSIM aims to simulate sea ice under all the variable conditions of the Arctic and Antarctic, our 1-D parametrization must be able to deal with a much wider range of changing boundary conditions. We adopt the Wells et al. [2010] 2-D results to a 1-D parametrization using the following assumptions:

[35] (1) If the Rayleigh number of a layer is above a critical value, brine leaves the ice via brine channels into the underlying ocean.

[36] (2) The amount of brine leaving each layer $i$ is proportional to $R^{i}-R_{\text {crit }}$.

[37] (3) All brine that leaves through channels is replaced by brine moving upward through the mush from the ocean. 


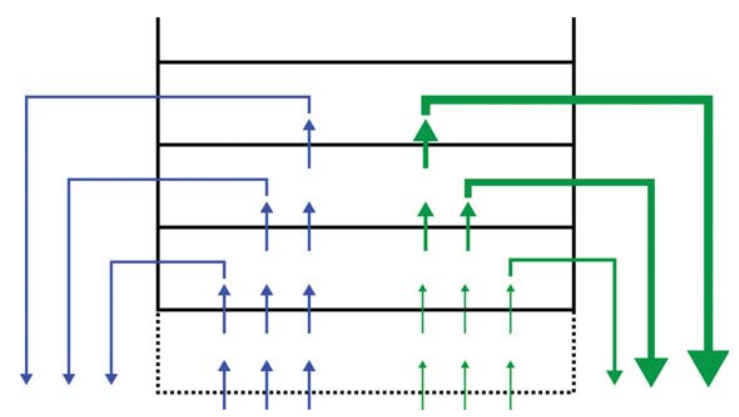

Figure 2. Sketch of brine fluxes (blue) and resulting salinity fluxes (green) of the convective gravity-drainage parametrization in the bottom ice layers during growth (see section 3.2). The blue arrows leaving the column represent brine leaving the ice through brine channels and entering the ocean. The short blue arrows represent the upwelling brine which replaces the brine leaving the ice. Arrow thickness indicates flux strength. Although the brine fluxes are of the same strength, the resulting salt flux is stronger from the colder upper layers as the brine salinity is higher.

[38] (4) Brine moving upward transports salt and thermal energy from layer to layer.

[39] (5) Brine leaving the sea ice downward through channels moves quickly enough that thermal interactions with the surrounding ice can be neglected.

[40] Although we have strong support for all of these assumptions from 2-D simulations and experiments, the more the conditions in the 1-D model differ from the conditions simulated by Wells et al. [2010], the less confident we are in our assumptions. This is especially relevant for deep convection in thick ice. The first assumption implies that brine channels always exist when the Rayleigh number exceeds the critical value. Although this can be safely assumed near the ice-ocean interface, we have no evidence this assumption is always valid in thick ice. Cole and Shapiro [1998] found that brine channels typically extended $30-50 \mathrm{~cm}$ into $1.4 \mathrm{~m}$ thick slices of first-year ice taken from two locations near Barrow. However, no channels were found that extended completely through the ice sheet. To truly validate or invalidate our assumption a much more thorough study of brine channels would be necessary.

[41] The second assumption results in two free parameters, the critical Rayleigh number $R_{c r i t}$ and a proportionality constant $\alpha$ which has the physical dimension of $\mathrm{kg} /\left(\mathrm{m}^{3} \mathrm{~s}\right)$. How we estimate these parameters is described in section 4. This second assumption is not identical to the findings of Wells et al. [2010], because Wells et al. [2010] linked the total brine flux to a nonlocal Rayleigh number, and we link the brine flux of each layer to a local Rayleigh number. As no data or theory exist on how gravity drainage interacts with entrapped gas bubbles, our gravity-drainage parametrization simply ignores the gas fraction.

[42] Assumptions three and four are similar to those of the channel-active-passive-zone model proposed by Rees Jones and Worster [2013] and are justified by the results of Wells et al. [2010] and Rees Jones and Worster (submitted manuscript, 2012). Figure 2 contains a sketch of the resulting brine and salt fluxes at the bottom of growing sea ice. In the sketch, the second to fourth lowest layers are equally unstable $\left(R^{n-1}=R^{n-2}=R^{n-3}>R_{\text {crit }}\right)$, which leads to identical mass fluxes. Although the brine fluxes are of the same strength, the resulting salt flux is stronger from the colder upper layers because the brine salinity is higher. The resulting heat fluxes would be opposite to the salt fluxes, with the warmer lower layers moving heat upward into the colder layers.

[43] The model calculates the temperature, volume fractions, and brine salinity of all active layers from 1 to $n$ at the beginning of each time step according to equations (1)(3). Using those values the Rayleigh number of each layer (besides the lowest) is calculated. If $R^{i}>R_{\text {crit }}$ we consider the layer $i$ convectively unstable. The mass of brine that flows from layer $i\left(b r_{\downarrow}^{i}\right)$ into the ocean in a time step of length $\mathrm{d} t$ is

$$
b r_{\downarrow}^{i}=\alpha\left(R^{i}-R_{c r i t}\right) \Delta z^{i} \cdot d t .
$$

[44] The downward-flowing brine is scaled by the time step $\mathrm{d} t$ and the layer thickness $\Delta z$ and has the temperature and salinity of the layer it originated from. Note that what we refer to as brine mass flow is synonymous with liquid mass flow. After $b r_{\downarrow}$ has been computed for all layers, the resulting upward brine fluxes from layer $i+1$ to layer $i$ resulting from mass conservation are

$$
b r_{\uparrow}^{i}=b r_{\uparrow}^{i-1}+b r_{\downarrow}^{i}=\sum_{k=1}^{k=i} b r_{\downarrow}^{k}
$$

[45] The amount of brine entering the layer $i$ from below is $b r_{\uparrow}^{i}$ which equals the sum of brine leaving that layer. Since we advect salt with the upstream method, the amount of salt that enters the layer $i$ per time step is $b r_{\uparrow}^{i} \cdot S_{b r}^{i+1}$ and the amount of salt leaving the layer $i$ is $\left(b r_{\uparrow}^{i-1}+b r_{\downarrow}^{i}\right) \cdot S_{b r}^{i}$. The resulting change in absolute salinity is

$$
\Delta S_{a b s}^{i}=b r_{\uparrow}^{i} \cdot S_{b r}^{i+1}-\left(b r_{\uparrow}^{i-1}+b r_{\downarrow}^{i}\right) \cdot S_{b r}^{i}=b r_{\uparrow}^{i} \cdot\left(S_{b r}^{i+1}-S_{b r}^{i}\right) .
$$

[46] An implication of these assumptions is that brine movement occurs in convectively stable layers when a higher layer is convectively unstable. The physical rational behind this is that preexisting brine channels through the stable layers allows brine from the unstable layers to flow downward without interacting with the stable layers. However, the resulting upward welling brine fluxes through the mush advect salt and heat. As long as the temperature decreases toward the ice surface, the upwelling brine leads to a desalination of the stable layers, which in turn reduces the convective instability of the layers above.

[47] This convective parametrization requires a small time step, especially since the bottom layers of SAMSIM are thin. In this paper the bottom layers vary from $2 \mathrm{~mm}$ to $5 \mathrm{~cm}$. A basic numerical rule of thumb for 1-D advection is that the distance traveled by the fluid per time step should not be larger than a tenth of the grid spacing. Translated to SAMSIM this rule states that the volume of brine moving from layer to layer per time step should not be larger than a tenth of the brine volume in those layers. The brine volume of each layer and the flow are extremely variable, so a 
small time step is necessary to avoid numerical instabilities. Although a simple flux correction is implemented to ensure that the salt advection remains positive definite, the computational cost of the stand-alone model is small enough that we can chose the time step to be as small as we need.

[48] Recently, a scheme which shares some of our assumptions was successfully implemented into the Los Alamos Sea Ice Model [Turner et al., 2013].

\subsection{Simple Parametrization}

[49] The convective parametrization is ill suited for earth system models as it requires a small time step to avoid instabilities. As an alternative, we propose a simpler parametrization as a tool to improve sea ice thermodynamics and salt release into the ocean for climate models. In this paper we refer to the simpler salinity parametrization as the simple parametrization and to the more complex parametrization that calculates brine fluxes as the convective parametrization.

[50] The simple parametrization is based on the assumption that convectively unstable layers lose salinity until they are stable. This assumption is a simplification of the convective parametrization in which convectively unstable layers lose salt through convection. Instead of losing salt via convection, the simple parametrization directly reduces the amount of salt in the layer until the layer is stable. The simple parametrization always produces a stable salinity profile, while the convective parametrization slowly evolves toward a stable salinity profile. Since the simple salinity parametrization does not determine any brine fluxes, it is of very limited use to model biogeochemistry in the ice.

[51] The first step of the simple parametrization is identical to the convective parametrization; the Rayleigh numbers of all layers are calculated. If the Rayleigh number is higher than the critical value, the layer is considered convectively unstable. But instead of calculating brine fluxes and resulting salt transport, in the simple parametrization, we reduce the salinity by a certain fraction. So if $R^{i}>R_{\text {crit }}$, then the salinity will be multiplied with a fixed constant $\gamma<1$ leading to $S_{a b s}^{i}$ in the following time step being $\gamma S_{a b s}^{i}$. The resulting parametrization is unconditionally stable and can be summarized in the following line:

$$
\text { If at time step t: } R^{i}>R_{\text {crit }} \quad \text { Then }: S_{a b s}^{i}{ }^{t+1}=\gamma \cdot S_{a b s}^{i}{ }^{t} \text {. }
$$

[52] Again we have a parametrization with two free parameters: $R_{\text {crit }}$ and $\gamma$. For $R_{\text {crit }}$ we use the same value as the convective parametrization. The parameter $\gamma$ must have a value between 0 and 1 . The closer $\gamma$ is to 1 the smoother the salinity evolution, but $\gamma$ must be small enough to ensure that the salinity decreases faster than the surrounding ice conditions evolve. The smaller the time step, the closer $\gamma$ can be to 1 . We recommend keeping $\gamma$ above 0.9 , as large jumps in salinity lead to sudden temperature changes. Our experience indicates that slight changes of $\gamma$ do not affect the results much.

[53] We expect the largest differences between the two schemes to occur when sea ice grows rapidly, because the simple parametrization forces the salinity profile into equilibrium much quicker than the brine circulation of the con- vective parametrization. Another difference is that a convectively stable layer below unstable layers can desalinate in the convective parametrization but not in the simple parametrization.

\subsection{Permeability}

[54] In porous media, permeability is part of the proportionality constant in Darcy's law which relates flow rate to a pressure gradient. In contrast to static materials (such as sandstone) the permeability of sea ice is continuously evolving and is affected by temperature, ice structure, salinity, and flow direction. Brine movement in sea ice causes heat and salt transport, which leads to a change in permeability, which in turn affects the brine movement. This behavior leads to highly nonlinear effects which can be exceedingly difficult to capture in numerical models.

[55] The permeability of sea ice is an extremely complex ongoing research topic which has been studied extensively [e.g., Petrich et al., 2006; Golden et al., 2007; Pringle et al., 2009; Büttner, 2011; Jones et al., 2012]. In SAMSIM we define permeability as an empirical function of the fluid volume fraction. This commonly used approach neglects the ice structure, which seems justified for our purposes because Gough et al. [2012] concluded that desalination processes are mostly unaffected by the ice structure.

[56] All tests in this paper were conducted using the relationship proposed by Freitag [1999]:

$$
\Pi^{i}\left(\phi_{l}^{i}\right)=10^{-17}\left(10^{3} \phi_{l}^{i}\right)^{3.1}
$$

[57] We believe this empirically derived relationship is similar enough to the $\Pi=\Pi_{0}\left(\phi_{l}\right)^{3}$ used by Wells et al. [2010] to avoid issues when transferring the results of Wells et al. [2010] to SAMSIM.

[58] At low liquid fractions sea ice can become impermeable, and $\phi_{\text {lcrit }}=0.05$ is often used as threshold value under which the remaining brine pockets are assumed to be isolated from each other [e.g., Golden et al., 1998; Petrich et al., 2006; Golden et al., 2007; Vancoppenolle et al., 2010]. As SAMSIM attempts to represent a spatial average of possibly highly heterogeneous sea ice, we believe that small permeabilities at low liquid fractions are justifiable. Also, if a low permeability results in a Rayleigh number below $R_{\text {crit }}$, our gravity-drainage parametrizations predict no changes. So as long as $R<R_{\text {crit }}$, it is irrelevant if the ice is truly impermeable or not. This does not change the fact that the gravity-drainage parametrizations react strongly to changes in the assumed permeability as it directly affects the Rayleigh number, and therefore also the values of $\alpha$ and $R_{\text {crit }}$.

\section{Parameter Estimation and Evaluation}

[59] The convective parametrization introduced in section 3.2 contains two free parameters, the dimensionless $R_{\text {crit }}$ and $\alpha$ with the physical dimension of $\mathrm{kg} /\left(\mathrm{m}^{3} \mathrm{~s}\right)$. In this section we detail how we derived $\alpha$ and $R_{\text {crit }}$ from laboratory salinity measurements, and how we determined that both parameters are independent of the vertical resolution of the model. 


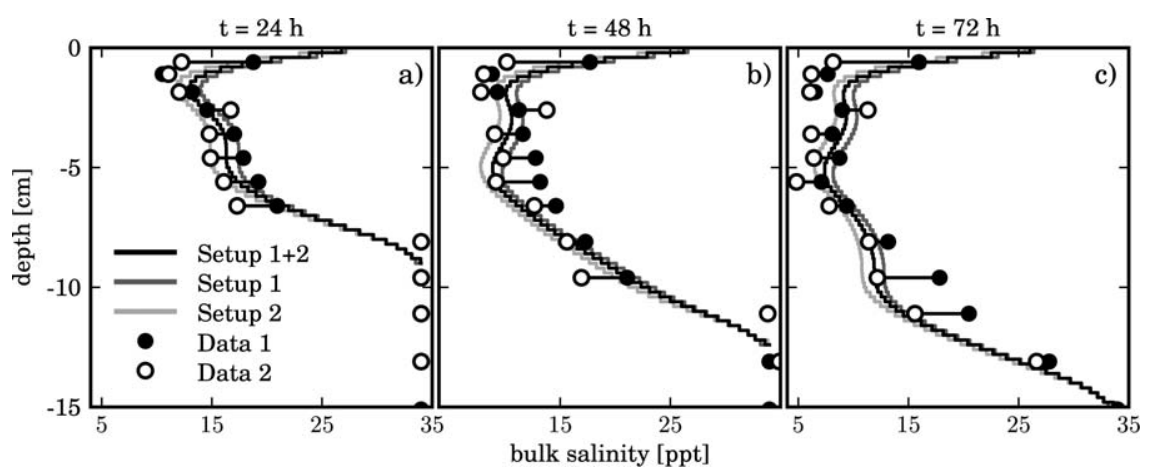

Figure 3. Bulk salinity measurements (dots) at different depths and corresponding model profiles at (a) $t=24 \mathrm{~h}$, (b) $t=48 \mathrm{~h}$, and (c) $t=72 \mathrm{~h}$. The free parameters of the gravity-drainage parametrization of setup 1 were optimized to fit data 1 , of setup 2 to fit data 2 , and of setup $1+2$ to fit the average of data 1 and data 2 . Grid parameters: $N=90, \Delta z_{0}=0.2 \mathrm{~cm}$. See section 4.1 for details on experimental setup and instrumentation.

\subsection{Salinity Measurements}

[60] The salinity measurements we use stem from a laboratory experiment that was described in section 8.4 of Notz [2005]. In this experiment, an $\mathrm{NaCl}$ solution was cooled from above by a cooling plate that was switched from $-5^{\circ} \mathrm{C}$ to $-10^{\circ} \mathrm{C}$ every $12 \mathrm{~h}$. The ice grew to almost $15 \mathrm{~cm}$ over the $72 \mathrm{~h}$ of the experiment, which was repeated once under identical conditions. Throughout the experiment, solid fraction and temperature were measured in situ at fixed depths at a high temporal resolution using a so-called wireharp [Notz and Worster, 2008]. The measured solid fraction is used to calculate the liquid fraction, and the brine salinity is derived from the measured temperature. Multiplying the liquid fraction with the brine salinity then allows us to calculate the bulk salinity.

[61] Although the precision of the wireharp was never determined thoroughly, tests with solid fractions below 0.8 agreed very well with theoretical expectations. However, at low liquid fractions slight measurement errors of the solid fraction lead to large errors in the bulk salinity. As a rule of thumb we assume that for liquid fractions above 0.2 the total error of bulk salinity is below $5 \mathrm{ppt}$, and that the relative error of bulk salinity increases with $1 / \phi_{l}$ as the liquid fraction approaches 0 .

[62] The three subpanels of Figure 3 show salinity measurements at three points in time. To what extent the differences between the two experiment repetitions (one marked by black dots and the other by white dots) are due to measurement errors or actual physical differences is impossible to tell. Cottier et al. [1999] showed that growing sea ice can have a high horizontal gradient in salinity linked to the location and morphology of the brine channels. We assume that the experiments were conducted under identical conditions, and the differences result from the sampling size of ice between the wires and measurement errors.

[63] We choose this experiment for multiple reasons. The first, and arguably the most important reason, is the high spatial resolution of the data. Also of great advantage is that the experiment was conducted twice, and that the controlled environment of the experiment can be easily translated to boundary conditions for the model. In contrast, field studies of sea ice contain many unknowns, such as precise heat fluxes and dynamic effects, which makes field measurements difficult to reproduce with a high degree of accuracy.

[64] Although a similar laboratory experiment was conducted with a fixed cooling temperature of $-10^{\circ} \mathrm{C}$, the results are unsuitable for a quantitative evaluation of the model. This results from of a combination of measurement uncertainty and the fact that the experiment was only conducted once. Additionally, inspecting the data uncovered multiple artifacts which further hinder a quantitative evaluation.

[65] The final reason for using this experiment is that the temperature of the cooling plate alternated between $-5^{\circ} \mathrm{C}$ and $-10^{\circ} \mathrm{C}$. Our convective parametrization is based on the results of Wells et al. [2010], in which a steady cooling temperature was assumed. If SAMSIM can reproduce the experiments, we have shown that our approach can deal with more complex conditions than those of Wells et al. [2010]. A limitation of the data is the rather short duration of the experiment. Also, the experiment would ideally have been conducted more than twice.

\subsection{Parameter Optimization}

[66] We use the Levenberg-Marquardt algorithm to determine the optimal values of $\alpha$ and $R_{\text {crit }}$ [Levenberg, 1944]. The metric which the algorithm seeks to minimize is the difference between the measured and the modeled salinity every $12 \mathrm{~h}$. If a measurement lies inside a model layer, it is directly compared to that layer. If the measurement lies between two layers, it is compared against the arithmetic mean of those two layers.

[67] We optimize the parameters separately for the first and second experiments measured by Notz [2005]. To ensure that the optimization results are not local minima, we chose four different initial estimations of $\alpha$ and $R_{\text {crit }}$. All four initial values result in almost identical values for all the data sets, which is by itself a promising sign (Figure 4a). The differences resulting from using other initial values are smaller than the precision criterion required to stop the algorithm. The two parameters vary by roughly a factor of 2 from set $1\left(\alpha=1.93 \times 10^{-3} \mathrm{~kg} /\left(\mathrm{m}^{3} \mathrm{~s}\right), R_{\text {crit }}=\right.$ $0.67)$ to set $2\left(\alpha=1.28 \times 10^{-3} \mathrm{~kg} /\left(\mathrm{m}^{3} \mathrm{~s}\right), R_{\text {crit }}=1.48\right)$. 


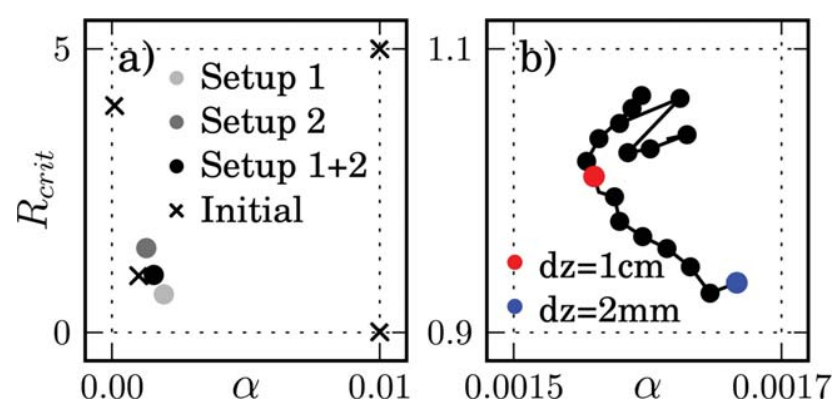

Figure 4. (a) Values of $R_{\text {crit }}$ and $\alpha$ derived by the Levenberg-Marquardt optimization algorithm for given sets of salinity measurements (" $1+2$ " is the average of sets " 1 " and " 2 "). For all initial parameter values (marked by an $\mathrm{x}$ ) the optimization results were identical. (b) Optimization results of $R_{c r i t}$ and $\alpha$ from a separate experiment for different vertical grid spacings $\mathrm{d} z ; \mathrm{d} z$ increases from 2 to $20 \mathrm{~mm}$ in $1 \mathrm{~mm}$ steps. Neighboring gird spacings (e.g., 3 and $4 \mathrm{~mm}$ ) are connected by a line. Note the different scales of Figures $4 a$ and $4 b$.

[68] To get an indication of how sensitive the optimization process reacts to small changes in the data, we create an additional artificial data set by averaging the two experiments (Figure 4a). Using this artificial data set the optimization process returns values which lie between the two previous results $\left(\alpha=1.56 \times 10^{-3} \mathrm{~kg} /\left(\mathrm{m}^{3} \mathrm{~s}\right), R_{\text {crit }}=1.01\right)$. We use these values as the default setting for SAMSIM.

\subsection{Resolution Dependency}

[69] To test the dependency of the parameters on the vertical resolution we conducted a simulation with a reference run at a vertical grid spacing of $1 \mathrm{~cm}$, in which ice was grown from a $\mathrm{NaCl}$ solution at a fixed cooling temperature over 6 days. A relatively high salinity of $70 \mathrm{ppt}$ was chosen to increase the strength of gravity drainage and the resulting freshwater signal. Every $12 \mathrm{~h}$ the freshwater content of all layers was saved. The Levenberg-Marquardt algorithm was used to optimize the model with different vertical resolutions to reach the same total freshwater content each $12 \mathrm{~h}$. In contrast to the previous subsection in which the salinity profile was used to determine the model performance, we choose to compare the freshwater content instead of comparing vertical profiles. This was done because comparing vertical profiles at different resolutions requires interpolation, and results would depend on the interpolation technique used. We also choose to keep the cooling temperature steady to ensure a linear temperature profile, which minimizes thermodynamic differences due to the changing resolution.

[70] The spacing of the model varied from $2 \mathrm{~mm}$ to $2 \mathrm{~cm}$ in steps of $1 \mathrm{~mm}$, a range that covers most of the values used in this paper. We find that the variations of $\alpha$ and $R_{\text {crit }}$ are smaller than $10 \%$ and show no trend (Figure $4 \mathrm{~b}$ ). From this, we conclude that our parameters $\alpha$ and $R_{\text {crit }}-$ which we determined using a $2 \mathrm{~mm}$ grid - do not seem to depend on resolution and are valid for vertical resolutions up to at least $2 \mathrm{~cm}$.

\subsection{Parametrization Evaluation}

[71] Although we have determined our free parameters by optimizing the model using salinity measurements, pa- rameter fitting only reduces the model error as much as the structure of the parametrization allows. To determine whether the parametrization can reproduce the measurements we compare the resulting salinity profiles of the model for the different values of $\alpha$ and $R_{\text {crit }}$ against the measurements (Figure 3). The model output generally agrees very well with measurements, with almost all deviations being smaller than the measured uncertainty. This good agreement indicates that the assumptions on which our convective parametrization is based yield an appropriate model. Additionally, SAMSIM proves itself capable of reproducing the thermodynamics of the experiment.

[72] We cannot verify if the high salinity values directly at the cooling plate predicted by the model occurred during the experiments. But it is to be expected that the ice crystal formation at the beginning of the experiment includes crystalline processes that cannot be captured using mushy-layer theory. It is also difficult to keep the cooling plate at a constant negative temperature when initially brought in contact with the $\mathrm{NaCl}$ solution because of the very rapid initial exchange of latent heat. The resulting initial temperature fluctuations are not included in the boundary conditions of the model simulations.

[73] It is remarkable that despite the rather large spread of $\alpha$ and $R_{\text {crit }}$ the model setups 1,2 , and $1+2$ behave very similarly. This similar behavior can be attributed to the fact that gravity drainage is a relatively stable process. Increased convection leads to increased salt loss, which results in lower liquid fractions and permeability, which in turn reduces convection. Slow convection leads to ice with a higher permeability, which leads to increased convection.

[74] Although we determined in the previous subsection that the optimal parameter estimates of $\alpha$ and $R_{\text {crit }}$ were insensitive to grid size, a further test is conducted to determine the sensitivity of the parametrization and the model to changes in vertical resolution. This test again uses halfdaily alternating temperatures of $-5^{\circ} \mathrm{C}$ and $-10^{\circ} \mathrm{C}$ as a boundary condition. Comparing the salinity profiles after 10 days for six different vertical resolutions against each other shows two very important results (Figure 5). First, the bulk salinity values change very little even though the vertical resolution changes by a factor of 32 . And second, the profiles change only very little once the vertical resolution is sufficient to fully resolve the curves of the salinity profile. This test further demonstrates that the convective gravity-drainage parametrization is insensitive to grid sizes.

[75] In conclusion, we derived estimations of $\alpha$ and $R_{\text {crit }}$ which are independent of grid resolution. More data from longer experiments are needed to further improve the estimations of $\alpha$ and $R_{\text {crit }}$, which are highly dependent on the assumed permeability. Using these values of $\alpha$ and $R_{\text {crit }}$ in the convective parametrization enables the model to reproduce measured salinity profiles.

\section{Idealized Tests}

[76] After developing, tuning, and evaluating our convective gravity-drainage parametrization with small-scale laboratory data, we now study gravity drainage under various idealized conditions. The tests with idealized boundary conditions are used to study the depth and strength of 


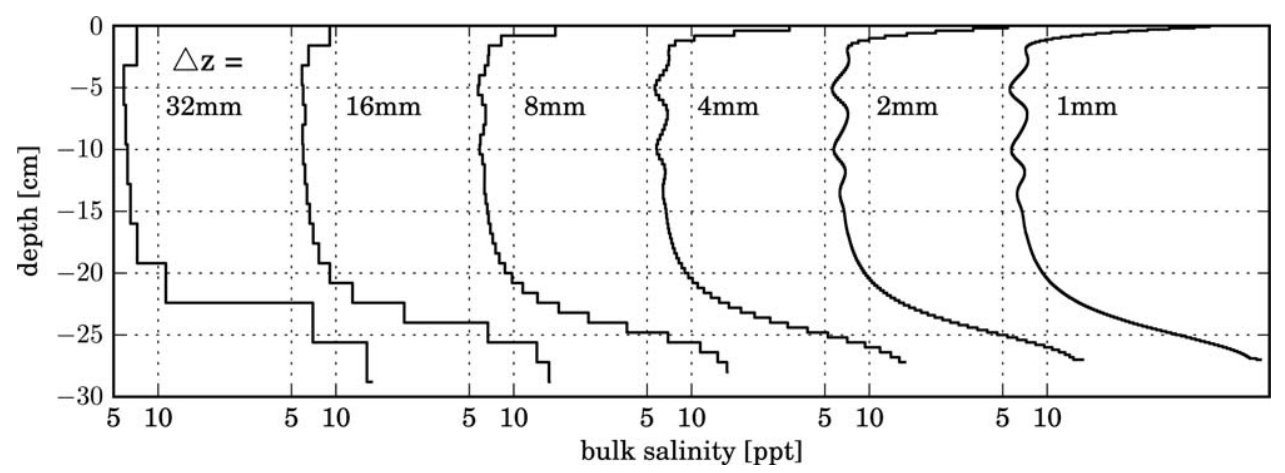

Figure 5. Bulk salinity model profiles for six different vertical resolutions after 10 days. Beware that the $x$ axis of each profile is shifted by $20 \mathrm{ppt}$ to improve visibility, i.e., the $5 \mathrm{ppt}$ line of the $16 \mathrm{~mm}$ profile is also the $25 \mathrm{ppt}$ line of the $32 \mathrm{~mm}$ profile. Grid parameters: $N=300, \Delta z_{0}=1-32 \mathrm{~mm}$.

gravity drainage, to quantify the desalination caused by gravity drainage, and to investigate the relationship between growth speed and the final bulk salinity of sea ice. The conclusions we draw from the idealized test cases are then tested under more realistic conditions in the next section, in which we force SAMSIM with reanalysis data.

\subsection{Constant Cooling}

[77] Our first test case is the freezing of a $\mathrm{NaCl}$ solution from a constant cooling temperature, which is the most often used setup for laboratory studies [e.g., Tait and Jaupart, 1992; Chen, 1995; Wettlaufer et al., 1997; Notz and Worster, 2009].

[78] We conduct simulations of a $\mathrm{NaCl}$ solution freezing at four different cooling temperatures ranging from $-5^{\circ} \mathrm{C}$ to $-35^{\circ} \mathrm{C}$ to cover the full range of growth speeds that occur in the Arctic and Antarctic. For these tests SAMSIM's grid is set to $N_{\text {top }}=5, N_{\text {mid }}=10, N_{\text {bot }}=10$, and $\Delta z_{0}=1.0 \mathrm{~cm}$ with a time step of $5 \mathrm{~s}$. We wait until the ice grows to a thickness of $50 \mathrm{~cm}$ and then compare the resulting profiles of salinity, solid fraction, and Rayleigh number. These test cases provide a frame of reference on how the bulk salinity of sea ice is related to growth speed.

[79] We find that more salt is retained in the ice the colder the cooling temperature (Figure 6a), as was also found in the laboratory experiments of Cox and Weeks [1975] and Wettlaufer et al. [1997]. Based on the salinity profiles, we conclude that a layer of growing sea ice cannot retain more than 10 ppt salt once convection has ceased.

[80] Despite the higher salinity, the colder experiments have a slightly higher solid fraction (Figure 6b). This can easily be understood: because the colder experiments have higher brine salinities, the solid fraction must be higher than in the warmer experiment to inhibit convection and retain salt. In all simulations, almost all the convection occurs in the lowest $10 \mathrm{~cm}$ regardless of growth speed (Figure 6c).

[81] The Rayleigh number of the slower-growing ice remains close to the critical value of 1.01 in the top $40 \mathrm{~cm}$ of the ice, while the faster-growing ice is more stable there. In contrast, the faster-growing ice is much more unstable in the lowest $10 \mathrm{~cm}$. All simulations remain slightly unstable in the top $5 \mathrm{~cm}$, driving a very weak circulation over the complete $50 \mathrm{~cm}$. Here we can see a clear signature of the semiadaptive grid, where the depth of the top instability is determined by the amount of thin top layers. This top instability is maintained by the constant surface temperature. Slight fluctuations of this temperature would remove the instability by first increasing the Rayleigh number and convection. The slight increase in convection would desalinate the lower layers enough so that when the top temperature returns to the initial value the reduced permeability would stabilize the flow.

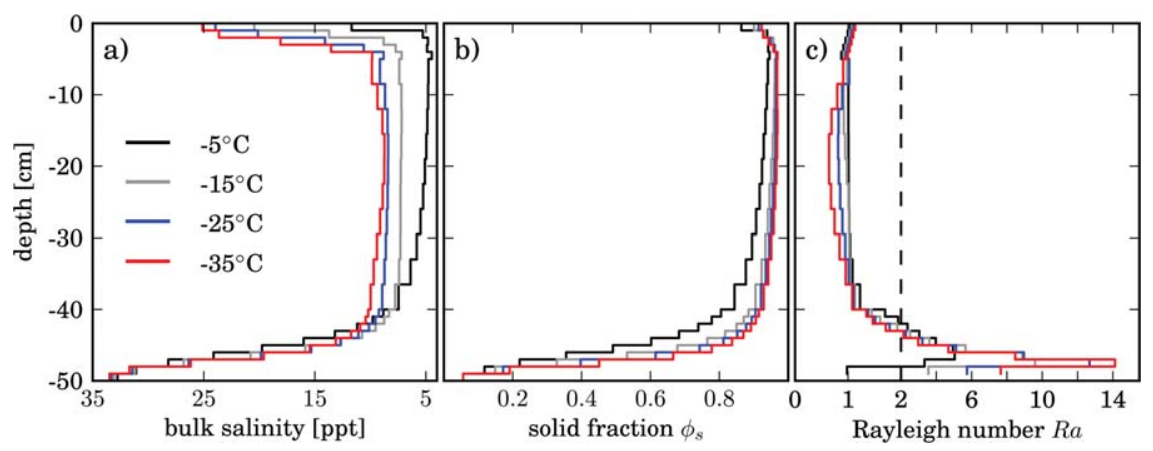

Figure 6. (a) Bulk salinity, (b) solid fraction, and (c) Rayleigh number profiles of freezing $\mathrm{NaCl}$ from a fixed surface temperature. Simulations were run until the ice thickness reached $50 \mathrm{~cm}$. Please notice that the scale of the $x$ axis in Figure $6 \mathrm{c}$ changes above 2 (marked by dashed line). All layers with $R$ greater than the critical Rayleigh number of 1.01 are convectively unstable. Grid parameters: $N=25, N_{\text {top }}=5, N_{\text {bot }}=10$, $\Delta z_{0}=1 \mathrm{~cm}$. 

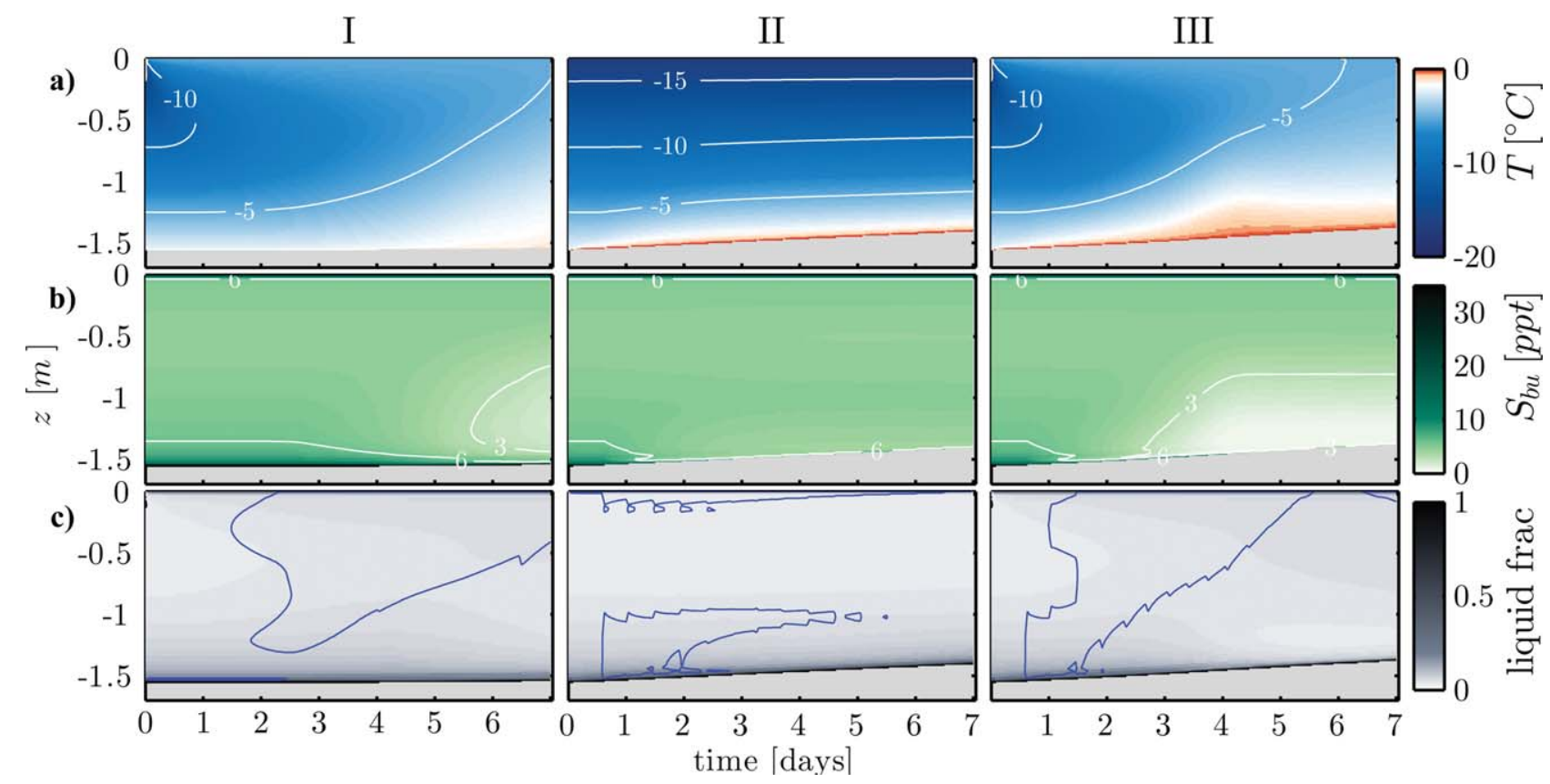

Figure 7. (a) Temperature, (b) bulk salinity, and (c) liquid volume fraction over 1 week. The blue line in row $\mathrm{C}$ encloses convectively unstable layers. Beginning from identical stable initial conditions: experiment I raises the top temperature from $-16.7^{\circ} \mathrm{C}$ to $-5^{\circ} \mathrm{C}$, experiment II increases the oceanic heat flux from 20 to $100 \mathrm{~W}$, and experiment III combines experiments I and II. Grid parameters: $N=70, N_{\text {top }}=5, N_{\text {bot }}=5$, $\Delta z_{0}=1 \mathrm{~cm}$.

[82] To summarize these results, we find that slowgrowing warm ice desalinates stronger and results in a marginally stable Rayleigh number profile. In contrast, faster-growing colder ice retains more salt, and the ice becomes convectively stable once gravity drainage ceases. As almost all convection occurs in the lowest $10 \mathrm{~cm}$, we conclude that multiple layers in the lowest $10-20 \mathrm{~cm}$ are necessary to properly simulate gravity drainage numerically. The relationship of higher salinity for fast growth speed and lower salinity for lower growth speed was also found in laboratory experiments by Cox and Weeks [1974]. These experiments were used to derive a fractionation coefficient based on growth velocity that describes the incorporation of salt into the advancing front. However, our results agree with the findings of Notz and Worster [2009] that such fractionation coefficient does not reflect the underlying physics of the measured relationship between growth speed and sea ice bulk salinity. We will further examine this relationship for more realistic boundary conditions in section 6.1 .

\subsection{Warming Triggered Convection}

[83] It is currently unclear if gravity drainage can occur in warming sea ice. Measurements of salt fluxes below sea ice [Widell et al., 2006] and of algae behavior in sea ice during autumn [Fritsen et al., 1994] indicate that convection may occur, as do recent observations of short-lived salinity anomalies under warming sea ice [Jardon et al., 2013]. In this subsection, we introduce an experiment designed to test if it is possible to trigger gravity drainage in sea ice by warming the ice from above and/or below. A secondary goal is to study how gravity drainage affects the sea ice.

[84] In principle, warming sea ice can lead to gravity drainage by increasing the permeability of the ice. Also, melting at the ice-ocean boundary can increase the buoyancy of the brine. The buoyancy is increased by the reduction of the salinity below the ice caused by melting ice at the ice-ocean boundary. Assuming the brine salinity in the sea ice remains steady, fresher water below the ice creates a larger density difference.

[85] To maximize our chance of triggering convection, we create initial conditions that are just stable. These initial conditions are reached by growing ice from a fixed temperature of $-16.7^{\circ} \mathrm{C}$ from salt water with a salinity of $34 \mathrm{ppt}$. The sea ice grows until it reaches a thickness at which the prescribed ocean heat flux of $20 \mathrm{~W} / \mathrm{m}^{2}$ balances the growth. Over the roughly 18 months simulated to reach the equilibrium state, gravity drainage slowly desalinates the ice until the Rayleigh numbers are just below the critical value.

[86] Three different experiments were applied using the stable initial conditions to trigger deep convection. Experiment I raises the top temperature from $-16.7^{\circ} \mathrm{C}$ to $-5^{\circ} \mathrm{C}$ to increase permeability while reducing buoyancy. Experiment II increases the oceanic heat flux from 20 to $100 \mathrm{~W}$ to increase buoyancy by melting ice at the ice-ocean boundary. Experiment III is a combination of the atmospheric and the oceanic forcing in experiments I and II.

[87] All three experiments succeeded in triggering convection in SAMSIM, with each experiment resulting in different convection patterns and salinity profiles (Figure 7). Experiment I mostly destabilizes the upper half of the ice (Figure $7 \mathrm{c}$, experiment I), but the strongest desalination occurs in the bottom half of the ice (Figure 7b, experiment I). The increased oceanic heat flux of forcing II destabilizes the lowest $50 \mathrm{~cm}$ and the top $10 \mathrm{~cm}$ (Figure $7 \mathrm{c}$, experiment II). The desalination caused in experiment II is weaker than the desalination of experiment I and is mostly confined to 


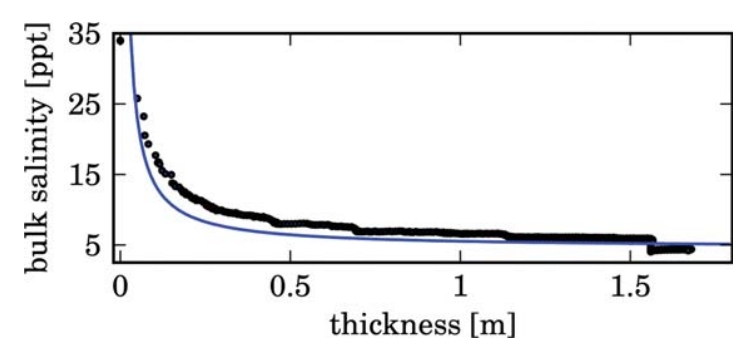

Figure 8. Reanalysis-forced daily model values of bulk salinity versus ice thickness (dots) and empirical relation of Kovacs [1997].

the lowest $40 \mathrm{~cm}$ (Figure 7b, experiment II). This desalination caused by an increased oceanic heat flux is possibly what was observed by Widell et al. [2006], who linked salt release to upward oceanic heat fluxes.

[88] The convection and desalination results of experiment III can be interpreted as an accelerated linear combination of the convection and desalination of experiments I and II. The resulting desalination is strong enough that it leads to a visible warming in the lower $40 \mathrm{~cm}$ after 4 days, as the ice solidifies and warms at the same time (Figure 7 , experiment III).

[89] From these three experiments we conclude, that gravity drainage can occur during top warming and bottom melt under ideal conditions. Warming the ice from above creates a stronger effect than melting the ice from below, and a combination of both leads to the strongest effects. In contrast to the gravity drainage that occurs during growth, the resulting deeper convection can span the whole ice layer. The desalination caused by the deep convection is strongest in the lower half for two reasons. First, as the amount of upwelling brine in each layer $n$ equals the sum of all brine flowing downward from above the layer $n$, the amount of upwelling is always largest in the lowest layer and decreases upward. Second, the temperature gradient is steeper in the lower than in the upper half due to the top warming. The combination of more upwelling and a higher gradient leads to a stronger salt advection, which results in a stronger desalination in the lower layers (equation (5)).

[90] In nature, atmospheric and oceanic forcing could easily be as strong or stronger than the idealized forcings we used in this experiment. However, it is highly unlikely that the initial ice conditions of the idealized experiments occur naturally. From these two statements we conclude that deep convection is possible in reality, but the resulting convection will likely be weaker than in the idealized experiments. The desalination of the lower half of sea ice after the onset of flushing, which was already noted by Malmgren [1927] and Holt and Digby [1985], could be the result of such warming-induced deep convection.

\section{Seasonal Growth Under Reanalysis Forcing}

[91] To examine how gravity drainage occurs under more realistic conditions, we conduct a case study of a single growth season using reanalysis data. We use this test case to determine which of our results from the idealized tests (such as those concerning deep convection and the link between growth speed and final salinity) are also valid under realistic conditions (section 6.1). This test case is also used to compare the simple against the convective gravity-drainage parametrization and to quantify the effect of gravity drainage on the thermal properties of sea ice (sections 6.2 and 6.3).

[92] To force SAMSIM with reanalysis data, the surface temperature is derived by balancing outgoing long-wave radiation with three-hourly ERA-interim fluxes. Both the fluxes and precipitation were taken from a grid point close to where the Surface Heat Budget of the Arctic Ocean (SHEBA) campaign was conducted [Perovich et al., 1999], namely, at $75^{\circ} \mathrm{N}$ and $217.5^{\circ} \mathrm{E}$. We randomly chose the year 2005 to simulate the total growth season from ice formation to maximum thickness. Snow accumulates over time in the single snow layer of variable thickness (see section 2.2). To avoid numerical instabilities the comparatively small heat flux into the snow and the sensible heat flux to the atmosphere are not included in the surface energy balance.

[93] As we have no reanalysis data of oceanic heat fluxes we approximate the oceanic heat flux as a simple sine curve with a period of 1 year, which is based loosely on the values Huwald et al. [2005b] derived from the SHEBA measurements. The oceanic heat flux reaches $14 \mathrm{~W} / \mathrm{m}^{2}$ in autumn and sinks to $0 \mathrm{~W} / \mathrm{m}^{2}$ in spring. For SAMSIM's grid we choose $N_{\text {top }}=10, N_{\text {mid }}=40, N_{\text {bot }}=20$, and $\Delta z_{0}=1.0 \mathrm{~cm}$ to highly resolve the bottom $20 \mathrm{~cm}$ of the ice. To avoid numerical issues in these small layers we use a time step of $10 \mathrm{~s}$. Aspects we neglect in this simulation are the initial formation of frazil ice and the feedbacks of the sea ice on oceanic, sensible, and latent heat fluxes.

[94] To determine if the model output is realistic, we evaluate the test case against data from the SHEBA Baltimore site and against the empirical relationship derived from over 400 Arctic ice cores by Kovacs [1997]. Our model produces a similar dependence of mean bulk salinity on ice thickness as given by the empirical function of Kovacs [1997] (Figure 8). In thin ice, modeled bulk salinity is slightly higher, which could also be related to the outflow of brine during sampling, which causes an underestimate of sampled bulk salinity in thin sea ice.

[95] A comparison with buoy data of first-year ice from the SHEBA Baltimore site (Perovich et al., 2012, Observing and understanding climate change: Monitoring the mass balance, motion, and thickness of arctic sea ice, http:// imb.crrel.usace.army.mil/) shows a good general agreement between simulated and measured temperature profiles (not shown). In the case study, the model grows $1.8 \mathrm{~m}$ of ice and accumulates approximately $30 \mathrm{~cm}$ of snow (Figure 9). The model is somewhat thicker than the maximum thickness of $1.5 \mathrm{~m}$ measured at the Baltimore site, but the Baltimore site is likely somewhat thinner due to the thicker snow cover of $50 \mathrm{~cm}$ compared to the $30 \mathrm{~cm}$ of snow in our case study. From the general similarities of the model with the SHEBA data and the empirical salinity-thickness relationship of Kovacs [1997] we conclude that the model results fulfill basic expectations.

\subsection{Gravity Drainage Under Reanalysis Forcing}

[96] The high spatial and temporal resolution of the case study simulation supplies a wealth of information on how gravity drainage, salinity, and temperature interact (Figure 9). From this data, we draw conclusions on the depth and variability of gravity drainage, the salinity evolution in 


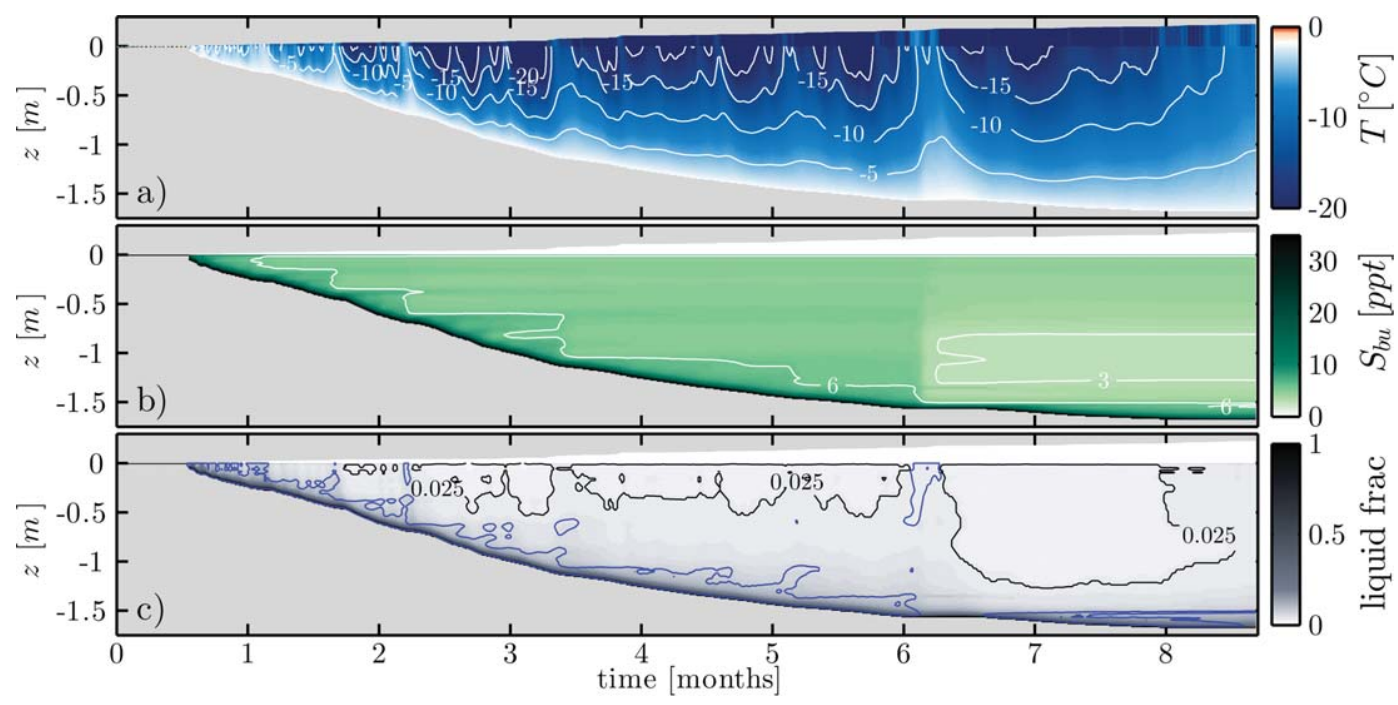

Figure 9. (a) Temperature, (b) bulk salinity, (c) and liquid volume fraction over a growth season (see section 6). In Figure $9 \mathrm{c}$ the blue line encloses convectively unstable layers, and the black line encloses regions with a liquid fraction below $2.5 \%$. The single snow layer on top of the sea ice lies above $z=0$. Grid parameters: $N=70, N_{\text {top }}=10, N_{\text {bot }}=20, \Delta z_{0}=1 \mathrm{~cm}$.

growing sea ice, how gravity drainage responds to temperature, and how salinity is linked to growth speed.

[97] From the blue line in Figure 9c we can see that although gravity drainage occurs mostly in the lowest $20 \mathrm{~cm}$, there is a great amount of variation. Not only does the convection depth at the bottom vary, but also additional layers separated from the lower convection become unstable now and then. Most notable is the full depth convection after 6 months when top warming destabilizes the top 50 $\mathrm{cm}$ of ice. Similar events of smaller magnitude occur shortly after 2 months and after roughly three and a half months. This variance of gravity drainage is not a simple reaction to temperature forcing or random model behavior. Instead, this variance results from the complicated interplay of salinity, buoyancy, and permeability.

[98] Comparing the $6 \mathrm{ppt}$ salt contour of Figure $9 \mathrm{~b}$ to the blue line of Figure 9c shows that the 6 ppt contour roughly outlines the lower convective ice layers. The $6 \mathrm{ppt}$ contour shows a stepwise shape at approximately 1.2, 1.6, 2.2, 3.3, 5.2, and 6 months. These steps all coincide with a warming of the ice, as can be seen in Figure 9a. At the same time, the depth of gravity drainage increases for a short time and then collapses. From this behavior, we conclude that gravity drainage reacts in cycles to the temperature evolution. The cycle begins when the surface temperature drops and

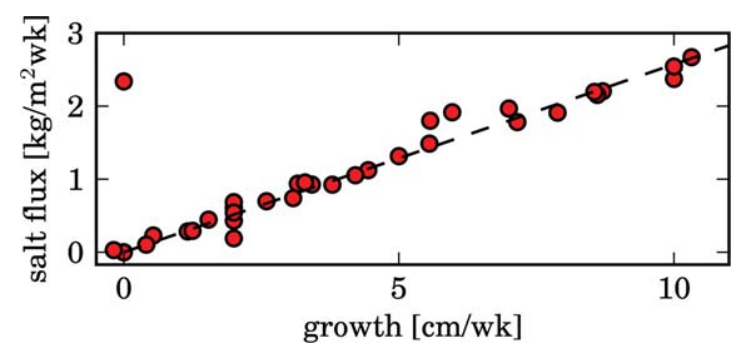

Figure 10. Reanalysis-forced weekly summed values of modeled salt flux versus growth speed. ice grows faster at the ice-ocean boundary. While the ice continues to grow, the newly formed ice remains convectively unstable. At some point in time the surface temperature rises again. As the ice warms, the convection depth increases or remains constant. When the ice once again begins to cool, most of the convectively unstable regions stabilize and the cycle repeats itself. Such a cycle in Figure 9 begins shortly before and ends slightly after 2 months, during which the top temperature drops from above $-5^{\circ} \mathrm{C}$ to below $-20^{\circ} \mathrm{C}$ and returns above $-10^{\circ} \mathrm{C}$. These cycles are visible as slight kinks or jumps when the bulk salinity is compared against the thickness (Figure 8).

[99] We will now turn to comparing these results to those from the idealized test case described in section 5. Doing so, it is interesting to note that in the simulation under realistic forcing, gravity drainage reduces the salinity to a stable value below $6 \mathrm{ppt}$. This value of $6 \mathrm{ppt}$ lies below the upper threshold of $10 \mathrm{ppt}$ which we determined from idealized experiments in section 5 to be the absolute maximum salinity possible in stable sea ice. In addition, the link between faster growth speed and higher salinity that we found in section 5 no longer holds: Such a link would result in a nonlinear relationship of salt flux to growth rate, which we do not find for the realistic forcing (Figure 10). The cyclic interaction of temperature and convection discussed in the above paragraph both disrupts the link between growth speed and salinity and causes a reduced stable bulk salinity in comparison to the experiments with a constant cooling temperature (Figure 6).

[100] In section 5 we concluded from idealized experiments that top warming can lead to gravity drainage over the whole ice layer. We also concluded that such convection would lead to a desalination which is strongest in the lower ice layers. The full depth convection that occurs after 6 months in the reanalysis-forced test case shows that both of these conclusions still hold for realistic boundary conditions. The resulting desalination is clearly visualized by the 3 ppt contour of salinity (Figure 9b). Comparing the $2.5 \%$ 


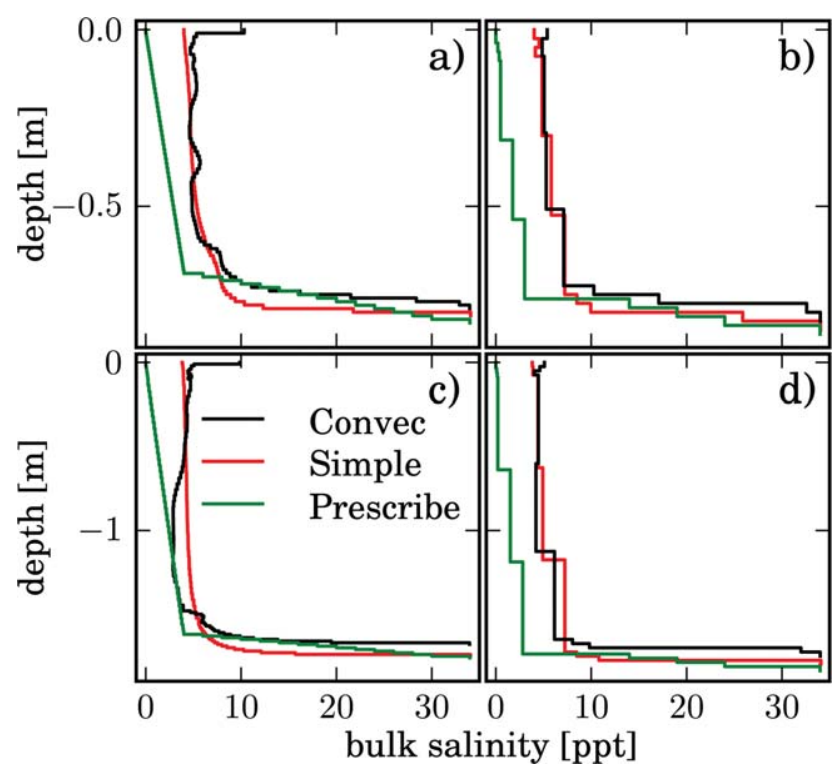

Figure 11. Case study salinity profiles of the convective and simple parametrization for two different vertical grids at two different times. The prescribed profile used in section 6.3 is also included. ( $\mathrm{a}$ and $\mathrm{b}$ ) $t \approx 3$ months and (c and d) $t \approx 9$ months. (Figures $11 \mathrm{a}$ and $11 \mathrm{c}$ ) Grid parameters: $N=70, N_{\text {top }}=5, N_{\text {bot }}=5, \Delta z_{0}=1 \mathrm{~cm}$. (Figures $11 \mathrm{~b}$ and 11d) Grid parameters: $N=10, N_{\text {top }}=3, N_{\text {bot }}=4$, $\Delta z_{0}=5 \mathrm{~cm}$.

contour (Figure 9c) before and after the event highlights the reduction in liquid fraction caused by the desalination.

\subsection{Convective Versus Simple Parametrization}

[101] To study how closely the simple parametrization (introduced in section 3.3) mimics the convective parametrization, we compare salinity profiles resulting from both parametrizations for the reanalysis-forced case study. As the simple parametrization is intended for use in coupled models in which using 70 levels is unthinkable, we also run SAMSIM at a lower resolution for this analysis. For the high-resolution case, we chose $N_{\text {top }}=10, N_{\text {mid }}=40$, $N_{b o t}=20$, and $\Delta z_{0}=1.0 \mathrm{~cm}$ (Figure 11a). The low resolution is based on $N_{\text {top }}=3, N_{\text {mid }}=3, N_{\text {bot }}=4$, and $\Delta z_{0}=$ $5 \mathrm{~cm}$ (Figure 11b).

[102] The simple convection provides a reasonable salinity profile approximation, especially at low resolution (Figures 11b and 11d). Although the convective parametrization desalinates growing sea ice somewhat slower, the differences are rather small. Two characteristics of the high-resolution convective parametrization are not reproduced by the simple parametrization: the high salinity in the top layer, and the desalination caused by deep convection after 6 months. The high salinity in the top layer cannot be reproduced by the simple parametrization because it has no sense of the speed of desalination and stabilizes the salinity profile almost immediately. The deep convection cannot be captured by the simple parametrization as it arises from the convective nature of gravity drainage.

\subsection{Relevance to Climate Models}

[103] In this subsection, we seek to quantify how relevant gravity drainage is for climate models. To achieve this, we compare the reanalysis-forced simulations using the convective and simple parametrization against simulations without gravity drainage. Due to their relevance in climate models we choose to evaluate ice thickness, enthalpy, thermal resistance, and freshwater column.

[104] The freshwater column describes the amount of freshwater contained in the sea ice and snow. It is calculated by melting the ice and snow and separating the resulting meltwater into freshwater and ocean water with a salinity of $34 \mathrm{ppt}$. For example, $2 \mathrm{~m}$ thick sea ice with a bulk salinity of $8 \mathrm{ppt}$ has a freshwater column of about $1.5 \mathrm{~m}$. The freshwater column grows when salt leaves sea ice and enters the ocean. Thermal resistance is the reciprocal of thermal conductance and is a measure of how strongly the sea ice resists the flow of heat between the ocean and atmosphere.

[105] The salinity of the comparison runs are determined by setting the salinity of the lowest layer where ice forms to 4 or 7 ppt, because these are plausible values of the bulk salinity of first-year ice. However, setting an initial salinity is not identical to the constant salinity approach often used in front-tracking models. The most significant difference is that in our model the freezing temperature at the ice-ocean interface is determined by the salinity of the water in the lowest layer into which the ice grows (4 or 7 ppt in this case), while front-tracking models can set the ice-ocean interface temperature independently of the sea ice and ocean salinity. Also, brine expulsion redistributes small amounts of salt.

[106] An additional comparison run is computed using a prescribed salinity profile which can be seen in Figure 11. In the lowest $15 \mathrm{~cm}$ the salinity decreases linearly from 34 too $4 \mathrm{ppt}$, and above that the salinity decreases to $0 \mathrm{ppt}$ at the surface. This setup is an imitation of prescribing a multiyear salinity profile in a front-tracking thermodynamic model, as is currently done in the Los Alamos Sea Ice Model. The brine fluxes from the ocean to the ice of the convective gravity drainage parametrization transport heat, which leads to a slight increase of oceanic heat flux. To ensure that the runs are comparable, the amount of heat transported by the moving brine is subtracted from the prescribed oceanic heat flux at each time step.

[107] In the rest of this subsection we will refer to the run using the full convective parametrization as the convec run, the run using the simple parametrization as the simple run, the run with the prescribed profile as prescribe, and the comparison runs as 4 and 7 ppt run in reference to their initial salinities. The same terminology is used in Figure 12.

[108] Vancoppenolle et al. [2006] conducted a similar experiment to quantify the effect of the full salinity evolution on the thermodynamic properties of the sea ice using a 1-D model with a parametrized salinity evolution. Although the aims of Vancoppenolle et al. [2006] were similar, our approaches differ in many crucial aspects detailed in Table 1 . We believe the most important differences are that Vancoppenolle et al. [2006] forced their runs with idealized climatological data, and that they ran their simulations until they reached an equilibrium, i.e., a constant annual cycle. Vancoppenolle et al. [2006] conclude that including a dynamic salinity component would significantly improve large-scale sea ice models. Vancoppenolle et al. [2009] studied the effects of adding a dynamic sea ice salinity component to the coupled NEMO-LIM3 ice-ocean model 

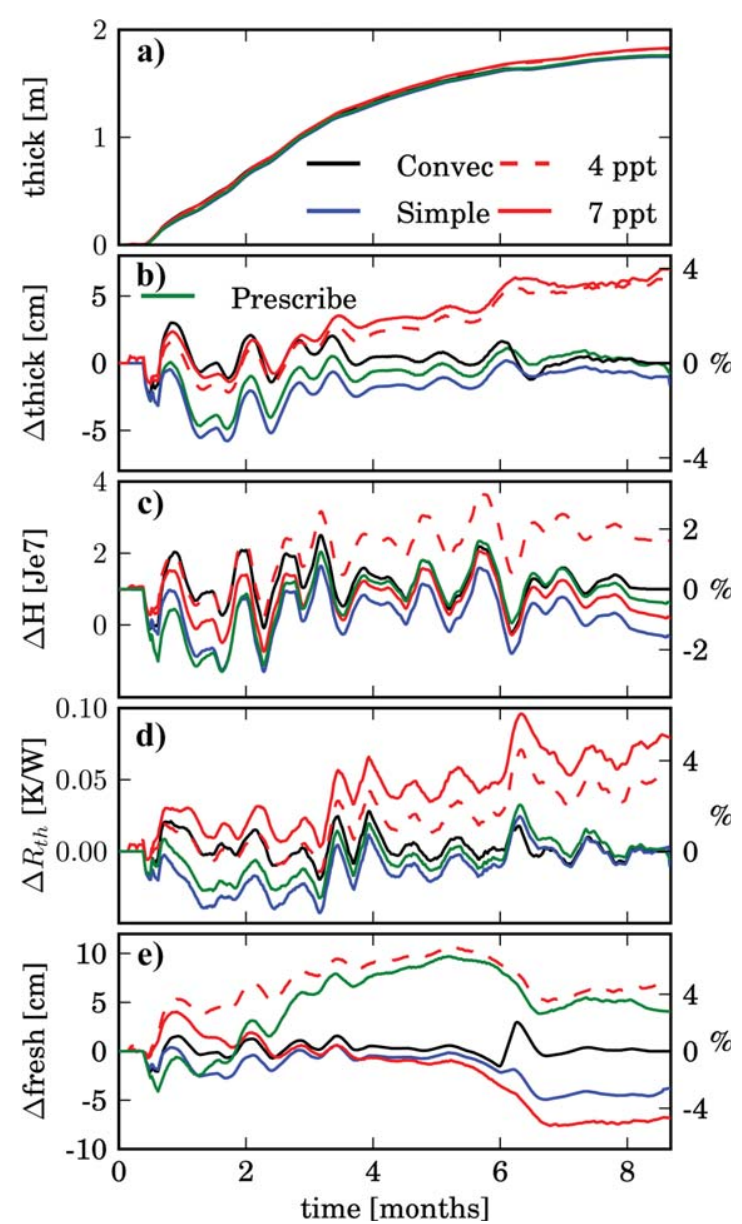

Figure 12. (a) Thickness, and differences of (b) thickness, (c) total enthalpy, (d) thermal resistance, and (e) fresh water column for the four salinity approaches. Convec, convective parametrization; Simple, simple parametrization; 4 ppt, initial salinity of $4 \mathrm{ppt;} 7 \mathrm{ppt}$, initial salinity of 7 ppt. Differences are calculated by first subtracting a moving monthly average of the convective parametrization and then applying a moving weekly average to reduce the noise. The percentages marked on the right $y$ axis of Figures $12 \mathrm{~b}-12 \mathrm{e}$ are the left $y$ axis values divided by the end values of "convec."

and concluded that the impact is similar to a $10 \%$ change of sea ice albedo, and advised accounting for varying sea ice salinity when simulating possible future climates.

[109] As expected, the 4 and 7 ppt runs produce thicker ice than the full convective run, in part owing to the higher freezing temperature and the higher thermal conductivity of fresher ice (Figure 12a). In the simulations with the simple parametrization and in those with a prescribed salinity profile, the ice grows slightly slower during the first month, but reaches the same thickness as simulated by the complex parametrization over the remaining 7 months.

[110] To study both short-term variations and long-term trends of the evaluated quantities, we subtract the running monthly mean of the convec run from all five runs. These differences are then smoothed by a weekly running average and plotted in Figures 12b-12e. The short-term variations of all runs agree well with two exceptions. The first excep- tion occurs during ice formation because the constant salinity runs freeze sooner and quicker. The second exception is visible in Figure 12e after 6 months when the deep convection occurs. Only the convec run shows a short-term freshening.

[111] At the end of the growth season the evaluated quantities of the simple run are $0-3 \%$ lower than the convec run. The prescribed and simple run are very similar, with the exception that the prescribed run has a higher freshwater column, which is reasonable since the prescribed profile is an approximation of a multiyear profile and saltless at the surface. Although the 4 and 7 ppt runs have a similar thickness, the 4 ppt run's thermal resistance is in better agreement with the convec run. In contrast, the enthalpy of the 7 ppt run agrees better than the 4 ppt run with the convec run. This shows that although the initial salinity can be varied to fit one quantity, no value can fit all. At the end of the growth season the average of all discrepancies over both the 4 and 7 ppt salinity runs is approximately 3\% compared to the convec run.

[112] In conclusion, in our test case the short-term variations of all approaches are similar, with the notable exception of the deep convection that only occurs in the simulation with the complex parametrization. The total effect of gravity drainage on the thermodynamic properties of the ice is rather modest, and differences between all runs and compared properties seldom exceed 4\% (Figure 12).

\section{Summary and Discussion}

\subsection{Summary}

[113] In this paper we have studied gravity drainage using a convective parametrization with two free parameters, namely, the critical Rayleigh number and a proportionality constant $\alpha$. Values for these two parameters were determined using the Levenberg-Marquadt optimization algorithm and salinity measurements from laboratory experiments. The optimization results were robust against changes in the initial values, but the uncertainties should be reduced with more data, especially from longer experiments. Our derived value of the critical Rayleigh number $(1 \pm 0.5)$ agrees well with theoretical expectations but is difficult to compare to the value of 5 used by Vancoppenolle et al. [2010] or the values of 0.5-2 which Gough et al.

Table 1. Comparison of Our Approach Against the Approach of Vancoppenolle et al. [2006] to Determine the Effect of the Salinity Evolution on the Thermal Properties of Sea Ice

\begin{tabular}{|c|c|c|}
\hline & This Study & $\begin{array}{c}\text { Vancoppenolle et al. } \\
{[2006]}\end{array}$ \\
\hline Forcing & Three-hourly reanalysis & Idealized climatological \\
\hline $\begin{array}{l}\text { Comparison } \\
\text { period }\end{array}$ & Growth season & $\begin{array}{l}\text { First-year and equilibrium } \\
\text { annual cycle }\end{array}$ \\
\hline Model & SAMSIM & Bitz and Lipscomb [1999] \\
\hline Vertical layers & 70 & $5-10$ \\
\hline Metrics & $\begin{array}{l}\text { Total enthalpy, thermal } \\
\text { resistivity freshwater } \\
\text { column }\end{array}$ & Thickness and salt flux \\
\hline $\begin{array}{l}\text { Desalination } \\
\text { processes } \\
\text { included }\end{array}$ & Gravity drainage & $\begin{array}{l}\text { Gravity drainage } \\
\text { and flushing }\end{array}$ \\
\hline
\end{tabular}


[2012] derived from ice-core measurements because slightly different definitions of the Rayleigh number were used. Vancoppenolle et al. [2010] and Gough et al. [2012] both use the thermal diffusivity of sea ice which is highly temperature- and salinity-dependent instead of the thermal diffusivity of brine we use in our definition of the Rayleigh number [Schwerdtfeger, 1963].

[114] The link between growth speed and resulting bulk salinity as indicated from laboratory experiments [Cox and Weeks, 1975; Wettlaufer et al., 1997] and field studies [Gough et al., 2012] is simulated by SAMSIM for sea ice growing from a fixed surface temperature. In contrast to the findings of these measurements, comparing salt release versus growth rate of a reanalysis-forced test case shows no indication that more salt is retained at faster growth speeds. In our model the strong temperature variations of the test case and the resulting destabilization of stable layers disrupt the link between growth speed and resulting bulk salinity.

[115] We show that SAMSIM allows for deep convection in sea ice, and deep convection can be found in both idealized and more realistic runs. The strongest salinity signal from deep convection is found in the lower and middle ice layers, which could explain observations of desalination near the ice-ocean interface during the melt season [Malmgren, 1927; Holt and Digby, 1985]. However, all results related to deep convection are somewhat speculative because deep convection is very sensitive to various model assumptions (e.g., permeability), and no direct measurements are available to compare SAMSIM's results against reality. We also show that under idealized conditions a freshening of the water directly under the ice caused by an increased oceanic heat flux can lead to gravity drainage near the ice-ocean interface. This mechanism could explain the link between salt flux and oceanic heat measured in the field by Widell et al. [2006].

[116] We compared a model run using the full convective gravity-drainage parametrization against runs with fixed salinities and showed that the total enthalpy, thermal resistance, and freshwater column differ over the growth season ( $\sim 3 \%$ ) but have similar short-term variations. Only at ice formation and during deep convection - the processes most difficult to reproduce correctly in a 1-D model-does model behavior diverge. As gravity drainage is the dominant but not sole desalination processes in sea ice, the effect of the total salinity evolution has yet to be assessed. Also, since the ocean and incoming atmospheric heat fluxes were prescribed, possible feedbacks were not included in this study.

[117] As a computationally cheap alternative to the convective parametrization we also developed an unconditionally stable and numerically cheap parametrization referred to as the simple parametrization. It is based on the assumption that the salinity profile evolves to reduce convective instability. The simple parametrization is capable of reproducing the general salinity profile of the convective parametrization and leads to an approximately $1 \%$ discrepancy of total enthalpy, thermal resistance, and freshwater column compared to the complex parametrization.

[118] An additional reference run was generated by prescribing a crude approximation of a multiyear salinity profile. The resulting thickness, total enthalpy, and thermal resistance evolution are very similar to those produced by the simple parametrization. However, the freshwater column is roughly $4 \%$ higher, which is to be expected since the prescribed profile is more similar to multiyear than first-year ice.

\subsection{Discussion}

[119] The convective parametrization of gravity drainage we presented provides a 1-D estimate of brine fluxes that is consistent with our physical understanding of the underlying processes. As such, it can aid researchers conducting detailed process studies of sea ice biogeochemistry and iceocean interaction.

[120] Our results provide insight into the relevance of gravity-drainage parameterizations for coupled climate models, but they cannot quantify the effect of the full salinity cycle including ice-ocean-atmosphere feedbacks. Given these limitations, our test case indicates that large-scale models would not profit greatly from the inclusion of a gravity-drainage parametrization. The complex parametrization is much too computationally demanding to be included in a large-scale model, and the effect on the thermal properties is rather small. The simple parametrization which was designed as a numerically effective alternative for large-scale models produces results achievable by directly prescribing a salinity profile. Especially for models with few layers (such as those proposed by Semtner [1976] and Winton [2000]) possible improvements are small compared to the overall model uncertainties [Wilkins, 2010].

[121] Acknowledgments. We would like to thank Juan Pedro Mellado, David Rees Jones, and our anonymous reviewers for helpful comments on previous versions which greatly improved this paper. Andrew Wells, Martin Vancoppenolle, and Christopher Petrich all provided ideas and advice during the early stages of our research for which we are grateful. We would also like to thank the ECMWF for providing ERA-interim reanalysis data and the University of Chicago for releasing MINPACK.

\section{References}

Bitz, C. M., and W. H. Lipscomb (1999), An energy-conserving thermodynamic model of sea ice, J. Geophys. Res., 104(C7), 15,669-15,677, doi:10.1029/1999JC900100.

Büttner, J. (2011), Permeability of young sea ice from microtomographic images, Master's thesis, Geophys. Inst., Univ. of Bergen, Bergen, Norway.

Chen, C. F. (1995), Experimental study of convection in a mushy layer during directional solidification, J. Fluid Mech., 293, 81-98, doi:10.1017/ S0022112095001649.

Chiareli, A. O. P., and M. G. Worster (1995), Flow focusing instability in a solidifying mushy layer, J. Fluid Mech., 297, 293-305, doi:10.1017/ S0022112095003107

Cole, D. M., and L. H. Shapiro (1998), Observations of brine drainage networks and microstructure of first-year sea ice, J. Geophys. Res., 103(C10), 21,739-21,750.

Cottier, F., H. Eicken, and P. Wadhams (1999), Linkages between salinity and brine channel distribution in young sea ice, J. Geophys. Res., 104(C7), 15,859-15,871, doi:10.1029/1999JC900128.

Cox, G., and W. Weeks (1975), Brine drainage and initial salt entrapment in sodium chloride ice, Tech. Rep. DTIC Doc.

Cox, G. F. N., and W. F. Weeks (1988), Numerical simulations of the profile properties of undeformed first-year sea ice during the growth season, J. Geophys. Res., 93(C10), 12,449-12,460, doi:10.1029/JC093iC10p 12449.

Feltham, D. L., N. Untersteiner, J. S. Wettlaufer, and M. G. Worster (2006), Sea ice is a mushy layer, Geophys. Res. Lett., 33, L14501, doi:10.1029/2006GL026290.

Freitag, J. (1999), The hydraulic properties of arctic sea ice-Implications for the small scale particle transport [in German], Ber. Polarforsch., 325, 17-84. 
Fritsen, C. H., V. I. Lytle, S. F. Ackley, and C. W. Sullivan (1994), Autumn bloom of Antarctic pack-ice algae, Science, 266(5186), 782-784.

Golden, K., S. Ackley, and V. Lytle (1998), The percolation phase transition in sea ice, Science, 282(5397), 2238-2241.

Golden, K. M., H. Eicken, A. L. Heaton, J. Miner, D. J. Pringle, and J. Zhu (2007), Thermal evolution of permeability and microstructure in sea ice, Geophys. Res. Lett., 34, L16501, doi:10.1029/2007GL030447.

Gough, A. J., A. R. Mahoney, P. J. Langhorne, M. J. M. Williams, and T. G. Haskell (2012), Sea ice salinity and structure: A winter time series of salinity and its distribution, J. Geophys. Res., 117, C03008, doi:10.1029/ 2011JC007527.

Gu, W., Y. Lin, Y. Xu, S. Yuan, J. Tao, L. Li, and C. Liu (2012), Sea ice desalination under the force of gravity in low temperature environments, Desalination, 295, 11-15.

Holt, B., and S. A. Digby (1985), Processes and imagery of first-year fast sea ice during the melt season, J. Geophys. Res., 90(C3), 5045-5062, doi: 10.1029/JC090iC03p05045.

Hunke, E. C., D. Notz, A. K. Turner, and M. Vancoppenolle (2011), The multiphase physics of sea ice: A review for model developers, Cryosphere, 5(4), 989-1009, doi:10.5194/tc-5-989-2011.

Huwald, H., L.-B. Tremblay, and H. Blatter (2005a), Reconciling different observational data sets from surface heat budget of the Arctic Ocean (SHEBA) for model validation purposes, J. Geophys. Res., 110, C05009, doi:10.1029/2003JC002221.

Huwald, H., L.-B. Tremblay, and H. Blatter (2005b), A multilayer sigmacoordinate thermodynamic sea ice model: Validation against surface heat budget of the Arctic Ocean (SHEBA)/sea ice model intercomparison project part 2 (SIMIP2) data, J. Geophys. Res., 110, C05010, doi: 10.1029/2004JC002328.

Jardon, F. P., F. Vivier, M. Vancoppenolle, A. Lourenco, P. Bouruet-Aubertot, and Y. Cuypers (2013), Full-depth desalination of warm sea ice, $J$. Geophys. Res. Oceans, 118, 435-447, doi:10.1029/2012JC007962.

Jeffery, N., E. C. Hunke, and S. M. Elliott (2011), Modeling the transport of passive tracers in sea ice, J. Geophys. Res., 116, C07020, doi:10.1029/2010JC006527.

Jones, K. A., M. Ingham, and H. Eicken (2012), Modeling the anisotropic brine microstructure in first-year arctic sea ice, J. Geophys. Res., 117, C02005, doi:10.1029/2011JC007607.

Kovacs, A. (1997), Sea ice. Part 1: Bulk salinity versus ice floe thickness, CRREL Rep. 96-7.

Lecomte, O., T. Fichefet, M. Vancoppenolle, and M. Nicolaus (2011), A new snow thermodynamic scheme for large-scale sea-ice models, Ann. Glaciol., 52(57), 337-346.

Lei, R., Z. Li, B. Cheng, Z. Zhang, and P. Heil (2010), Annual cycle of landfast sea ice in Prydz Bay, east Antarctica, J. Geophys. Res., 115, C02006, doi:10.1029/2008JC005223.

Levenberg, K. (1944), A method for the solution of certain non-linear problems in least squares, Q. J. Appl. Math., II(2), 164-168.

Maksym, T., and M. O. Jeffries (2000), A one-dimensional percolation model of flooding and snow ice formation on Antarctic sea ice, J. Geophys. Res., 105(C11), 26,313-26,331, doi:10.1029/2000JC900130.

Malmgren, F. (1927), On the properties of sea-ice, in Norwegian North Polar Expedition with the MAUD, 1918-1925, Scientific Results, Geophys. Inst., Bergen, Norway.

Massonnet, F., T. Fichefet, H. Goosse, M. Vancoppenolle, P. Mathiot, and C. K. Beatty (2011), On the influence of model physics on simulations of Arctic and Antarctic sea ice, Cryosphere, 5(3), 687-699, doi:10.5194/tc5-687-2011.

Maykut, G. A., and N. Untersteiner (1971), Some results from a timedependent thermodynamic model of sea ice, J. Geophys. Res., 76(6), 1550-1575, doi:10.1029/JC076i006p01550.

Nakawo, M., and N. K. Sinha (1981), Growth-rate and salinity profile of 1st-year sea ice in the high arctic, J. Glaciol., 27(96), 315-330.

Notz, D. (2005), Thermodynamic and fluid-dynamical processes in sea ice, $\mathrm{PhD}$ thesis, Univ. of Cambridge, Cambridge, U. K.

Notz, D., and M. G. Worster (2006), A one-dimensional enthalpy model of sea ice, Ann. Glaciol., 44(1), 123-128, doi:10.3189/172756406781811196.

Notz, D., and M. G. Worster (2008), In situ measurements of the evolution of young sea ice, J. Geophys. Res., 113, C03001, doi:10.1029/2007J C004333.
Notz, D., and M. G. Worster (2009), Desalination processes of sea ice revisited, J. Geophys. Res., 114, C05006, doi:10.1029/2008JC 004885 .

Oertling, A. B., and R. G. Watts (2004), Growth of and brine drainage from $\mathrm{NaCl}-\mathrm{H}_{2} \mathrm{O}$ freezing: A simulation of young sea ice, J. Geophys. Res., 109, C04013, doi:10.1029/2001JC001109.

Perovich, D. K., et al. (1999), Year on ice gives climate insights, Eos Trans. $A G U, 80(41), 481-486$, doi:10.1029/EO080i041p00481-01.

Petrich, C., P. J. Langhorne, and Z. F. Sun (2004), Numerical simulation of sea ice growth and desalination, in Proceedings of the 17 th International Symposium on Ice, vol. 3, pp. 68-78, International Association of Hydraulic Engineering and Research, Saint Petersburg, Russia.

Petrich, C., P. J. Langhorne, and Z. F. Sun (2006), Modelling the interrelationships between permeability, effective porosity and total porosity in sea ice, Cold Reg. Sci. Technol., 44(2), 131-144, doi:10.1016/j.coldre gions.2005.10.001.

Pringle, D. J., J. E. Miner, H. Eicken, and K. M. Golden (2009), Pore space percolation in sea ice single crystals, J. Geophys. Res., 114, C12017, doi:10.1029/2008JC005145.

Rees Jones, D. J., and M. G. Worster (2013), A simple dynamical model for gravity drainage of brine from growing sea ice, Geophys. Res. Lett., 40, 307-311, doi:10.1029/2012GL054301.

Roscoe, H. K., B. Brooks, A. V. Jackson, M. H. Smith, S. J. Walker, R. W. Obbard, and E. W. Wolff (2011), Frost flowers in the laboratory: Growth, characteristics, aerosol, and the underlying sea ice, J. Geophys. Res., 116, D12301, doi:10.1029/2010JD015144.

Saenz, B. T., and K. R. Arrigo (2012), Simulation of a sea ice ecosystem using a hybrid model for slush layer desalination, J. Geophys. Res., 117, C05007, doi:10.1029/2011JC007544.

Schwerdtfeger, P. (1963), The thermal properties of sea ice, J. Glaciol., 4(36), 789-807.

Semtner, A. J. (1976), Model for thermodynamic growth of sea ice in numerical investigations of climate, J. Phys. Oceanogr., 6(3), 379-389.

Tait, S., and C. Jaupart (1992), Compositional convection in a reactive crystalline mush and melt differentiation, J. Geophys. Res., 97(B5), 6735-6756.

Turner, A. K., E. C. Hunke, and C. M. Bitz (2013), Two modes of sea-ice gravity drainage: A parametrization for large-scale modeling, J. Geophys. Res., doi:10.1002/jgrc.20171, in press.

Vancoppenolle, M., T. Fichefet, and C. M. Bitz (2006), Modeling the salinity profile of undeformed Arctic sea ice, Geophys. Res. Lett., 33, L21501, doi:10.1029/2006GL028342.

Vancoppenolle, M., T. Fichefet, and H. Goosse (2009), Simulating the mass balance and salinity of Arctic and Antarctic sea ice: 2. Importance of sea ice salinity variations, Ocean Modell., 27, 54-69, doi:10.1016/ j.ocemod.2008.11.003.

Vancoppenolle, M., H. Goosse, A. de Montety, T. Fichefet, B. Tremblay, and J.-L. Tison (2010), Modeling brine and nutrient dynamics in Antarctic sea ice: The case of dissolved silica, J. Geophys. Res., 115, C02005, doi:10.1029/2009JC005369.

Wells, A. J., J. S. Wettlaufer, and S. A. Orszag (2010), Maximal potential energy transport: A variational principle for solidification problems, Phys. Rev. Lett., 105(25), 254502, doi:10.1103/PhysRevLett.105. 254502.

Wells, A. J., J. S. Wettlaufer, and S. A. Orszag (2011), Brine fluxes from growing sea ice, Geophys. Res. Lett., 38, L04501, doi:10.1029/2010GL 046288 .

Wettlaufer, J. S., M. G. Worster, and H. E. Huppert (1997), Natural convection during solidification of an alloy from above with application to the evolution of sea ice, J. Fluid Mech., 344, 291-316, doi:10.1017/ S0022112097006022.

Widell, K., I. Fer, and P. M. Haugan (2006), Salt release from warming sea ice RID g-2701-2010, Geophys. Res. Lett., 33, L12501, doi:10.1029/ 2006 GL026262.

Wilkins, N. (2010), Thermodynamics of thin sea ice, $\mathrm{PhD}$ thesis, Meteorol. Inst., Univ. of Hamburg, Hamburg, Germany.

Winton, M. (2000), A reformulated three-layer sea ice model, J. Atmos. Oceanic Technol., 17(4), 525-531. 